\title{
1 A protocol for genome-wide analysis of DNA replication timing in intact root tips
}

2 Running head: DNA replication timing in intact root tips

3

4 Leigh Mickelson-Young ${ }^{1}$ (co first author), Emily E. Wear ${ }^{1}$ (co first author), Jawon Song ${ }^{2}$,

5 Gregory J. Zynda ${ }^{2}$, Linda Hanley-Bowdoin ${ }^{1}$, and William F. Thompson ${ }^{1}$

$7 \quad{ }^{1}$ Department of Plant and Microbial Biology, North Carolina State University, Raleigh, NC

827695 and $^{2}$ Texas Advanced Computing Center, University of Texas, Austin, Texas 78758

9

10 Corresponding Author:

11 Leigh Mickelson-Young

12 email:lamickel@ncsu.edu

\section{Abstract}

15 DNA replication during S phase in eukaryotes is a highly-regulated process that ensures the

16 accurate transmission of genetic material to daughter cells during cell division. Replication

17 follows a well-defined temporal program, which has been studied extensively in humans,

18 Drosophila, and yeast, where it is clear that the replication process is both temporally and

19 spatially ordered. The replication timing (RT) program is increasingly considered to be a

20 functional read out of genomic features and chromatin organization. Although there is increasing

21 evidence that plants display important differences in their DNA replication process compared to

22 animals, RT programs in plants have not been extensively studied. To address this deficiency, we 
23 developed an improved protocol for the genome-wide RT analysis by sequencing newly

24 replicated DNA ("Repli-seq") and applied it to the characterization of RT in maize root tips. Our

25 protocol uses 5-ethynyl-2'-deoxyuridine (EdU) to label replicating DNA in vivo in intact roots.

26 Our protocol also eliminates the need for synchronization and frequently associated chemical

27 perturbations as well as the need for cell cultures, which can accumulate genetic and epigenetic

28 differences over time. EdU can be fluorescently labeled under mild conditions and does not

29 degrade sub-nuclear structure, allowing for the differentiation of labeled and unlabeled nuclei by

30 flow sorting, effectively eliminating contamination issues that can result from sorting on DNA

31 content alone. We also developed an analysis pipeline for analyzing and classifying regions of

32 replication and present it in a point-and-click application called Repliscan that eliminates the

33 need for command line programming.

34

35 Key words 5-ethynyl-2'-deoxyuridine, cell cycle, DNA replication, flow cytometry, Repli-seq,

36 replication timing, maize, plant

37

38

39 The temporal program of DNA replication along the chromosomes in eukaryotes is a highly

40 regulated and reproducible process (1) that ensures the accurate transmission of genetic material

41 to each daughter cell during cell division. DNA replication timing (RT) studies have revealed

42 that replication is a temporally ordered process that is coordinated with transcription, chromatin

43 dynamics, and the three-dimensional chromatin landscape $(2,3)$. For example, early replicating

44 DNA is transcriptionally active, rich in genes and contains euchromatic histone modifications (4-

45 10), while late replicating DNA is associated with heterochromatin and repetitive elements (5,

46 11). Furthermore, chromosome conformation capture studies revealed that early and late 
47 replication strongly correlates with open and closed chromatin compartments, respectively (12-

48 14). Increasingly, replication time is thought to be an important functional readout of genomic

49 features and chromatin organization (15).

50 The information we have on RT programs comes mostly from fungal and metazoan systems (2,

51 16) and, until recently, very little was known about the RT programs in plants. Although the

52 replication machinery is broadly conserved across eukaryotes, there are numerous differences

53 between plants and other eukaryotes. For example, plants lack geminin $(17,18)$ and Rif1 $(19$,

54 20), two proteins intimately involved in the regulation of DNA replication. Plants also display

55 differences in the spatiotemporal distribution of initiation regions (21) and of replicating DNA

56 within the nucleus $(22,23)$. Moreover, S-phase duration, on average, is shorter in plants than that

57 of mammals (24), indicating that plant replication programs may be temporally compressed

58 compared to those of animals. These differences emphasize the importance of studying DNA

59 replication in plants, as replication programs are likely to differ between kingdoms and phyla,

60 and information specific to replication in plant systems is needed to integrate with data on

61 chromatin dynamics and gene expression.

62 Our group has recently characterized RT programs in maize and Arabidopsis (21, 25-28). In this

63 chapter, we present a Repli-seq protocol (Fig. 1) that we developed to characterize DNA

64 replication timing (RT) programs in maize root tips. Although our system is conceptually similar

65 to the Repli-seq protocol first described for human cells (8), our system takes advantage of a new

66 labeling method that allows the physical separation of labeled and unlabeled nuclei. We used this

67 protocol to provide the first whole genome analysis of RT in a higher plant (26), and to extend

68 our original analysis of Arabidopsis chromosome 4 (25) to the entire genome (27). Additionally,

69 we present a computational pipeline called Repliscan (29) that we developed to analyze 
70 sequencing data generated by the Repli-seq protocol, and present it here as a "point-and-click"

71 application hosted on CyVerse.

73 Our protocol uses 5-ethynyl-2'-deoxyuridine (EdU) to label replicating DNA in vivo for $20 \mathrm{~min}$

74 in the maize root tip. After labeling, we dissect the $0-1 \mathrm{~mm}$ portion of the root which contains

75 the meristem where many cells undergo a conventional mitotic cell cycle (30). Dissected root

76 tips are fixed, and nuclei are bulk isolated by a method modified from the original chopping

77 method of Galbraith and colleagues $(25,31,32)$. Incorporated EdU is conjugated in situ to

78 Alexa fluor (AF) 488 using click chemistry (33). Nuclei are counterstained with 4,6-diamidino-

79 2-phenylindole (DAPI) and fractionated into different portions of S phase using two color flow

80 cytometry and sorting. EdU-labeled nuclei are cleanly separated from unlabeled G1 and G2

81 nuclei, and divided into early, middle, and late S-phase populations based on DNA content. DNA

82 is extracted from the early, middle, and late fractions, and the EdU-labeled DNA is

83 immunoprecipitated (IP) using an anti AF-488 antibody. IP DNA is sequenced, the sequencing

84 reads are mapped to the maize B73 reference genome, and analysis using Repliscan classifies the

85 B73 genome by replication time (26).

87 A major improvement of our Repli-seq protocol, as compared to the first reported protocol (8), is

88 that we use the thymidine analog, EdU, instead of 5-bromo-2'-deoxyuridine (BrdU) to label

89 replicating DNA in vivo. Visualization of EdU using click chemistry (33) is achieved rapidly and

90 under mild conditions that do not degrade nuclei or subnuclear structure (34), in contrast to the

91 harsh conditions needed to visualize BrdU by immunocytochemistry. As noted above, EdU 
92 labeling also permits intact, replicating nuclei (S-phase nuclei containing EdU) to be

93 unambiguously distinguished from non-replicating nuclei (G1 and G2 nuclei that do not

94 incorporate EdU) by flow cytometry. Our protocol thus provides pure populations of labeled,

95 replicating nuclei, which cannot be achieved when sorting based solely on DNA content (26, 32).

96 The additional purification reduces the contamination of unlabeled DNA during

97 immunoprecipitations. The improved separation is especially important in the early and late S-

98 phase fractions, which otherwise would contain a large excess of unlabeled nuclei (Fig. 2c, d).

100 Another advantage of our protocol is that labeling occurs in an intact organ. Maize root tips

101 contain many proliferating cells that efficiently label with EdU and can be easily isolated for

102 analysis (32). EdU has also been used successfully to label DNA in other plant systems,

103 including plant cell cultures $(24,30)$. The protocol is easily applied to unsynchronized cell

104 populations, as occurs in root tips, avoiding disruptive synchronization treatments such as

105 application of chemical inhibitors and sucrose starvation (35-37). RT analysis of intact root tips

106 also avoids any genetic or epigenetic instability that can occur in plant cell cultures (38-40).

107 Although, at this time, we cannot separate different cell types of the root tip, our analysis

108 pipeline identified RT differences between mitotic vs endocycling cells, suggesting possible RT

109 differences among cell types (28).

111 One further benefit of this protocol is that we highlight the Repliscan pipeline that we developed 112 to detect and classify regions of replication into discrete combinations of replication signatures.

113 The utility, accuracy and general applicability of Repliscan has been validated through reanalysis 
114 of existing BrdU-based, Repli-seq data from human and Drosophila (29). The point-and-click

115 format of the Repliscan application presented in this protocol facilitates the production of

116 accurate RT profiles without the need for command line programming. These RT profiles can be

117 compared to other genomic features (i.e. genes, GC content, nuclease sensitivity and

118 transcriptional activity) (26).

119 The steps needed for successful production and analysis of quality Repli-seq data for maize are 120 presented below. This protocol can be easily adapted to other plant systems.

\section{2. Materials}

123 Wear the appropriate personal protective equipment when handling reagents that pose any hazard 124 and dispose according to local regulations. All chemicals used are reagent grade. The following 125 are supplies and equipment that are repeated and needed at various times throughout the 126 protocol.

127 Supplies: 2-mL round-bottom microcentrifuge tubes, 5-mL round-bottom polypropylene tubes, 128 microscope slides, Qubit dsDNA High Sensitivity Assay Kit and tubes.

129 Equipment: refrigerated microcentrifuge, Qubit fluorometer, refrigerator or cold room, heated 130 water baths or incubators, and fluorescence microscope.

\subsection{Plant material and growing conditions}

133 1. Zea mays cv B73 seeds. Approximately $450-550$ seeds per experiment based on an $80 \%$ 134 germination rate.

1352 2. $150-250 \mathrm{~mL} 10 \%$ commercial bleach solution containing $0.05 \%$ Tween 20 

glass pyrex dish, Magenta boxes, paper towels

\subsection{In vivo EdU pulse-labelling of replicating DNA, dissecting and fixing root tips}

1. $40 \mathrm{mM}$ stock solution of 5-ethynyl-2'-deoxyuridine (EdU) in dimethyl sulfoxide

\subsection{Isolating nuclei} (DMSO), stored at $-80^{\circ} \mathrm{C}$.

2. $1 \times$ Phosphate buffered saline (PBS)

3. $16 \%$ Paraformaldehyde (EM grade)

4. 2 M Glycine, filter sterilized

5. Liquid nitrogen

6. Supplies: large rectangular dish (similar to dimensions 7 in. long by 4 in. wide by 4 in. deep), \#10 scalpel, fine tipped forceps (curved and tapered similar to Fisher \#12-000131), petri dishes, small glass beaker (25-50 mL), Kimwipes

7. Equipment: orbital shaker, vacuum pump attached to a desiccator

1. Cell lysis buffer (CLB): $15 \mathrm{mM}$ Tris (pH 7.5), 2 mM Na 2 EDTA, $80 \mathrm{mM} \mathrm{KCl,} 20 \mathrm{mM}$ $\mathrm{NaCl}, 0.1 \%$ Triton $\mathrm{X}-100$. Adjust $\mathrm{pH}$ to 7.5 and then add $15 \mathrm{mM}$ 2-mercaptoethanol (buffer modified from LB01 in (41)).

2. cOmplete ${ }^{\mathrm{TM}}$ or cOmplete ${ }^{\mathrm{TM}}$ Mini protease inhibitor tablets (Roche)

3. Supplies: Miracloth, small plastic funnel, $50 \mathrm{~mL}$ conical tubes 
4. Equipment: commercial food processor (like Cuisinart ${ }^{\circledR}$ Mini-Prep ${ }^{\circledR}$ 2.6-cup food processor, model DLC-1SS); refrigerated swing bucket centrifuge with rotor to hold 50 $\mathrm{mL}$ tubes

\subsection{Clicking EdU to Alexa fluor 488}

1. Click-iT® EdU Alexa Fluor ${ }^{\circledR} 488$ Imaging Kit (Life Technologies)

2. Modified CLB: $15 \mathrm{mM}$ Tris (pH 7.5), $80 \mathrm{mM} \mathrm{KCl,} 20 \mathrm{mM} \mathrm{NaCl}, 0.1 \%$ Triton X-100, pH 7.5

3. 4,6-diamidino-2-phenylindole (DAPI) stock solution: $1 \mathrm{mg} / \mathrm{mL}$ in sterile $\mathrm{H}_{2} \mathrm{O}$

4. CLB containing $2 \mu \mathrm{g} / \mathrm{mL}$ DAPI

5. CellTrics 20- $\mu \mathrm{m}$ nylon mesh filters (Partec)

\subsection{Flow sorting of nuclei}

1. $1 \times$ Sodium chloride-Tris-Na $\mathrm{EDTA}_{2}$ (STE) buffer: $10 \mathrm{mM}$ Tris-HCl $(\mathrm{pH} 7.5), 1 \mathrm{mM}$ $\mathrm{Na}_{2} \mathrm{EDTA}(\mathrm{pH}$ 8), $100 \mathrm{mM} \mathrm{NaCl}, \mathrm{pH} 7.5$

2. Laser alignment beads suitable for the flow sorter and particle size to be analyzed. For the BD InFlux 3-3.4 $\mu \mathrm{m}$ and 8.1-12 $\mu \mathrm{m}$ Ultra Rainbow Fluorescent Beads (Spherotech) are one option.

3. Equipment: flow sorter equipped with a 355-nm UV laser and a 488-nm sapphire laser

\subsection{Reversing formaldehyde crosslinks and extracting genomic DNA}

1. $1 \times$ Sodium chloride-Tris-Na $\mathrm{NaDTA}_{2}$ STE) buffer: $10 \mathrm{mM}$ Tris- $\mathrm{HCl}(\mathrm{pH} 7.5), 1 \mathrm{mM}$ $\mathrm{Na}_{2} \operatorname{EDTA}(\mathrm{pH}$ 8), $100 \mathrm{mM} \mathrm{NaCl}, \mathrm{pH} 7.5$

2. $0.5 \mathrm{M} \mathrm{Na}_{2}$ EDTA, $\mathrm{pH} 8$

3. $30 \%$ Sodium lauroyl sarcosine 
4. Proteinase K (typically $23-27 \mathrm{mg} / \mathrm{mL}$ stock)

5. Phase lock gel tubes (2-mL tubes; for example, Quantabio 5PRIME Phase Lock Gel ${ }^{\mathrm{TM}}$ Light)

6. Phenol:chloroform:isoamyl alcohol (25:24:1 saturated with $10 \mathrm{mM}$ Tris $(\mathrm{pH} 8), 1 \mathrm{mM}$ $\mathrm{Na}_{2}$ EDTA). Phenol should be fresh and not oxidized.

7. Chloroform:isoamyl alcohol (24:1)

8. GlycoBlue (15 mg/mL stock)

9. $3 \mathrm{M}$ sodium acetate $(\mathrm{NaOAc}), \mathrm{pH} 7$

10. 2-propanol, molecular biology grade (Sigma \#I9516) stored at $-20^{\circ} \mathrm{C}$

\subsection{DNA shearing and immunoprecipitating Alexa fluor 488 clicked to EdU-labeled DNA}

The reagents are adapted from Gendrel et al. 2005 (42).

1. Chromatin immunoprecipitation (ChIP) dilution buffer: $16.7 \mathrm{mM}$ Tris (pH 8), $1.2 \mathrm{mM}$ $\mathrm{Na}_{2}$ EDTA, $167 \mathrm{mM} \mathrm{NaCl}, 1.1 \%$ Triton X-100

2. Dynabeads ${ }^{\circledR}$ Protein $G$ magnetic beads

3. Anti-Alexa Fluor 488 antibody (rabbit IgG, Molecular Probes, A-11094)

4. Low-salt wash: $20 \mathrm{mM}$ Tris- $\mathrm{HCl}(\mathrm{pH} 8), 2 \mathrm{mM} \mathrm{Na}{ }_{2}$ EDTA, $150 \mathrm{mM} \mathrm{NaCl}, 0.1 \%$ Sodium dodecyl sulfate (SDS), 1\% Triton X-100

5. High-salt wash: $20 \mathrm{mM}$ Tris- $\mathrm{HCl}(\mathrm{pH}$ 8), $2 \mathrm{mM} \mathrm{Na} 2$ EDTA, $500 \mathrm{mM} \mathrm{NaCl}, 0.1 \%$ Sodium dodecyl sulfate (SDS), $1 \%$ Triton X-100 
6. Lithium chloride (LiCl) wash: $10 \mathrm{mM}$ Tris- $\mathrm{HCl}(\mathrm{pH} 8), 1 \mathrm{mM} \mathrm{Na} \mathrm{EDDA}_{2}$, $250 \mathrm{mM} \mathrm{LiCl}$, 1\% Nonidet P-40 (NP-40) (or IGEPAL® CA-630, Sigma, which is chemically indistinguishable from NP-40), 1\% sodium deoxycholate

7. Tris, $\mathrm{Na}_{2} \mathrm{EDTA}$ (TE) buffer: $10 \mathrm{mM}$ Tris- $\mathrm{HCl}(\mathrm{pH} 8), 1 \mathrm{mM} \mathrm{Na}_{2} \mathrm{EDTA}$

8. Elution buffer: $1 \%$ sodium dodecyl sulfate (SDS), $100 \mathrm{mM}$ sodium bicarbonate

205 $\left(\mathrm{NaHCO}_{3}\right)$

9. $1 \mathrm{M}$ Tris, $\mathrm{pH} 6.5$

\subsection{Purifying immunoprecipitated DNA}

\subsection{Preparing the DNA sequencing library}

1. New England BioLabs ${ }^{\circledR}$ Inc. (NEB) NEBNext ${ }^{\circledR}$ Ultra II DNA library preparation kit 
3. $80 \% \mathrm{EtOH}$

4. NEBNext ${ }^{\circledR}$ Multiplex Oligos for Illumina ${ }^{\circledR}$ (96 Unique Dual Index Pairs, NEB \#E6640S)

225

5. Agilent Bioanalyzer High Sensitivity DNA Kit and High Sensitivity DNA chips

226

227

\subsection{Quality control, alignment and filtering of sequencing data}

1. CyVerse cyberinfrastucture account, register at http://user.cyverse.org.

2. Sequence files in FASTQ format, including single or paired-end reads. For maize, pairedend reads are highly recommended.

3. Reference genome, for example, Zea mays B73 RefGen_v4 available from the CyVerse Data Store accessible from the Discovery Environment (/iplant/home/shared/iplantcollaborative/genomeservices/builds/1.0.0/24_77/Zea.AGPv4/ de_support).

4. Analysis software: Table 1 lists the software that will be used in this chapter.

\subsection{Analyzing and classifying replication time using the Repliscan application}

1. A genome browser, like Integrative Genomics Viewer (IGV), available as a free download or web-based application (http://software.broadinstitute.org/software/igv/).

\section{Methods}




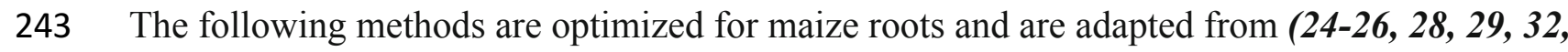

244 42).

245

246

247

\subsection{Plant material and growing conditions}

1. Imbibe 450-550 maize seeds overnight in a container filled with deionized $\mathrm{H}_{2} \mathrm{O}$. Place on a magnetic stir plate and add a stir bar to keep water constantly moving, and provide aeration with a fish tank bubbler.

2. The next day, drain the water and surface sterilize the imbibed seeds with a $10 \%$ commercial bleach solution containing $0.05 \%$ Tween 20 for 15 min with rotary mixing. Rinse the seeds three or four times with sterile $\mathrm{H}_{2} \mathrm{O}$.

3. Place 12 seeds in autoclaved magenta boxes pre-equipped with several layers of paper towels. Just prior to adding the seeds, dampen the paper towels with $10 \mathrm{~mL}$ sterile $\mathrm{H}_{2} \mathrm{O}$. Germinate the seeds for 3 days in a growth chamber set to $28^{\circ} \mathrm{C}$ with continuous, dim $\operatorname{light}(\sim 500$ lux $)$.

4. Repeat this process on different days for a total of three biological replicates.

\subsection{In vivo EdU pulse-labelling of replicating DNA, dissecting and fixing root tips}

$$
\text { Prepare the following prior to the labeling step: } 25 \mu \mathrm{M} \text { EdU solution in water from the } 40
$$
$\mathrm{mM}$ stock solution, $1 \times \mathrm{PBS}$ and $16 \%$ paraformaldehyde in $1 \times \mathrm{PBS}$.

1. Pour $\sim 300 \mathrm{~mL}$ of sterile $\mathrm{H}_{2} \mathrm{O}$ in a large rectangular dish (described in Materials). To rinse the roots, place the maize seedlings in the dish in such a way as to keep the roots immersed in the water as much as possible. The volume of water will depend on the number of seedlings and the size of the container. We describe what we typically use for $\sim 500$ maize seedlings. 
2. To label nascent DNA, pour off the rinse water and add $\sim 300 \mathrm{~mL}$ sterile $\mathrm{H}_{2} \mathrm{O}$ containing a final concentration of $25 \mu \mathrm{M}$ EdU (see Note 1), making sure the roots are immersed in the EdU solution. Incubate the seedlings for the desired labeling time (see Note 1, Fig. 3) with gentle shaking $(65 \mathrm{rpm})$ on a bench-top orbital shaker at room temperature in the dark.

3. Pour off the EdU solution and rinse the seedlings three times with at least $300 \mathrm{~mL}$ sterile $\mathrm{H}_{2} \mathrm{O}$ (1-2 volumes of EdU labeling solution). Leave some of the last rinse in the container to keep the roots moist. Avoid letting the root tips dry out.

4. Dissect the desired root tip segment (see Note 2) in a petri dish using a scalpel and finetipped forceps. In maize, we dissect the terminal $0-1 \mathrm{~mm}$ portion of the root tip.

5. Minimize the length of time for dissecting root segments as much as possible to avoid over fixation (see Note 3). Transfer the dissected segments to a small beaker or tube 5 min under vacuum. containing $20 \mathrm{~mL}$ of $1 \%$ paraformaldehyde and fix for $15 \mathrm{~min}$, with the first $5 \mathrm{~min}$ under vacuum.

6. Add $2 \mathrm{M}$ glycine to a final concentration $0.125 \mathrm{M}$ to quench the fixation, and incubate for

7. Remove the fixative from the root segments and wash them three times with $\sim 20 \mathrm{~mL} 1 \times$ PBS and remove the liquid completely with a pipet after the final wash. Transfer the root segments to a $2-\mathrm{mL}$ tube. Tap the root tips to the bottom of the tube and spin very lightly on a small table top centrifuge to bring any remaining liquid to the bottom of the tube. Remove the residual liquid with a $10-\mu \mathrm{L}$ pipet or by twisting a Kimwipe into a fine tip and carefully wicking away the last drop of liquid. It is important to remove as much 
liquid as possible to minimize the number of ice crystals formed during the freezing process. Snap-freeze the tubes in liquid nitrogen and store at $-80^{\circ} \mathrm{C}$.

290

291

292

293

294

295

296

297

298

299

300

301

302

303

\subsection{Isolating nuclei}

Nuclei are isolated from fixed, frozen root tip tissue by chopping the frozen pellet in a food

processor in cold CLB. All steps should be done on ice or at $4^{\circ} \mathrm{C}$. Additionally, the nuclei must be resuspended very well after every centrifugation to break up aggregates of nuclei and debris (See (32) for detailed blending optimization).

1. Prior to beginning, prepare fresh CLB and keep on ice. Chill the food processor, a swinging bucket centrifuge and a microcentrifuge in a cold room. If using refrigerated centrifuges, set to $4^{\circ} \mathrm{C}$.

2. If the nuclei preparation is done the day before the sorting experiment, supplement the CLB buffer with a Complete or Complete Mini protease inhibitor cocktail tablet by first dissolving the protease inhibitor tablet in the water used to make the buffer. Dissolving the tablet first prevents the buffer detergent from excessive foaming while mixing in the tablet (32).

3. Add 30-35 mL of chilled CLB to the food processor. Take the tube of root tip tissue out of the $-80^{\circ} \mathrm{C}$ freezer, and tap or flick the tube until the tissue pellet is released from the bottom. Transfer the pellet into the food processor with the buffer and chop at low speed for $15 \mathrm{sec}$ at a time with intervening 15 -sec breaks to reduce foaming. Do this $4-5$ times.

4. Allow the cellular homogenate to incubate in the food processor for $5 \mathrm{~min}$ at $4^{\circ} \mathrm{C}$ to facilitate nuclei release from the cells. Half way through this incubation period, gently swirl the homogenate to further aid the release of nuclei. 
5. Set up a double layer of Miracloth in the top of a small plastic funnel. Place the funnel apparatus over a 50-mL conical tube pre-chilled on ice. Pre-wet the Miracloth by adding 5-10 $\mathrm{mL}$ of CLB to the funnel apparatus and discard the flow through.

6. Swirl the homogenate one more time, and then filter it through the Miracloth into the tube. Allow the homogenate to filter through for several minutes, typically $5 \mathrm{~min}$, and

9. Proceed immediately to the click reaction (section 3.4).

\section{$325 \quad 3.4$ Clicking EdU to Alexa fluor 488}

326 The CLB buffer contains 2-mercaptoethanol and $\mathrm{Na}_{2}$ EDTA, both of which will inhibit the 327 copper mediated click reaction. Hence, for the click reaction to proceed efficiently, the nuclei 328 must be washed with a modified CLB buffer without 2-mercaptoethanol or $\mathrm{Na}_{2} \mathrm{EDTA}$. After 329 each addition of a buffer solution to the nuclei pellet, it is important to resuspend the nuclei 330 thoroughly to prevent them from clumping. Unless otherwise noted, resuspension is done by 331 pipetting up and down 50-100× using a 1-mL pipet, using a gentle action to minimize foaming.

332 All centrifugations are done at $200 \mathrm{xg}$ in a refrigerated microcentrifuge at $4^{\circ} \mathrm{C}$. Prior to 
333 beginning, prepare the following working and stock solutions of the Click-iT ${ }^{\circledR}$ EdU Alexa

334 Fluor ${ }^{\circledR} 488$ Imaging Kit: Alexa Fluor® 488 azide, $1 \times$ Click-iT® EdU reaction buffer, and 10×

335 Click-iT® EdU buffer additive, according to kit instructions.

336 1. Add $2 \mathrm{~mL}$ of modified CLB to each nuclei pellet and resuspend as described above.

337 Transfer the resuspended nuclei into $2-\mathrm{mL}$ round-bottomed microcentrifuge tubes.

338 2. Centrifuge for $5 \mathrm{~min}$ at $4^{\circ} \mathrm{C}$ to pellet nuclei. Remove the supernatant with a $1-\mathrm{mL}$ pipet 339 and immediately place the tube with the nuclei on ice.

3403 . During the above centrifugation step, prepare the Click-iT ${ }^{\circledR}$ reaction cocktail according 341 to the kit instructions.

342 4. Determine empirically the volume of Click-iT® reaction cocktail (see Note 4) to add and 343 resuspend the nuclei. We typically use $2 \mathrm{~mL}$ to resuspend nuclei from $\sim 500$ seedlings. To 344 minimize exposure to light, mix by pipetting up and down $\sim 25 \times$ and incubate for 30 min 345 at room temperature in the dark. The reaction can proceed on ice but is more efficient at 346 room temperature. Centrifuge for $5 \mathrm{~min}$ at $4^{\circ} \mathrm{C}$ to pellet the nuclei and discard the 347 supernatant.

348 5. Depending on the amount of Click-iT® reaction cocktail used, add twice the volume of 349 CLB to wash out the residual reaction cocktail, and resuspend the nuclei by pipetting up 350 and down $\sim 50 \times$. Centrifuge and discard the supernatant.

6. To stain total DNA, resuspend the nuclei pellet in an appropriate volume of CLB-DAPI (see Note 5) depending on nuclei yield. Pipet the sample as normal and allow at least 5 min for DAPI staining.

354 7. Check a small aliquot, e.g. $2.5-3.0 \mu \mathrm{L}$, of the nuclei suspension on a fluorescence 355 microscope to verify nuclei quality, density of the suspension, and DAPI and AF-488 
fluorescence. All of the nuclei should fluoresce with DAPI and only S-phase nuclei that incorporated EdU and were successfully clicked will fluoresce with AF-488.

8. If desired, store the nuclei overnight at $4^{\circ} \mathrm{C}$, protected from light.

9. Just prior to flow cytometric analysis and sorting, filter the nuclei suspension through a $20-\mu \mathrm{m}$ nylon mesh filter into a 5-mL round-bottom, polypropylene tube to remove large debris and nuclear aggregates.

\section{2}

\subsection{Flow sorting of nuclei}

363 What follows is a very basic description of flow cytometry and sorting. We recommend the 364 following references for more detailed descriptions: (43-48) and references therein.

Flow sorting, a combination of flow cytometry and sorting, measures the light scattering and

367 fluorescence properties of individual particles from a heterogeneous suspension and sorts them

368 on the basis of these measurements. Scattered light from particles crossing the laser beam or

369 "interrogation point" is measured as forward light scatter (FSC) and side scatter (SSC), which

370 provide information about the relative size and complexity (granularity and internal structure) of

371 the particle, respectively. We use combinations of FSC, SSC and fluorescence intensity to locate

372 nuclei of interest while excluding debris and broken nuclei from our sorted populations.

374 The captured light is detected by photomultiplier tubes (PMTs) which convert it to a voltage 375 pulse known as an "event". Pulse height (H), pulse width (W), and pulse area (A) will differ for 376 different particles (see $(46,49)$ for detailed description). Additionally, a "trigger" is used to

377 exclude or "threshold out" particles that do not meet a certain threshold amount of light

378 scattering or fluorescence. Various flow sorters will have different options for this parameter, so 
379 the trigger will need to be determined empirically for your sorter (see (46) for detailed

380 description). We use FSC as a trigger to threshold out signals that don't meet certain relative size

381 criteria, thus removing things like pulses of stray light, dust, debris, and broken nuclei.

383 The excitation and emission properties of a fluorescent particle must be compatible with the 384 cytometer's lasers and filters. In our experiments, nuclei are stained with DAPI and EdU is 385 conjugated to AF-488. DAPI fluorescence is excited by a 350-nm laser, and AF-488

386 fluorescence by a 460-nm laser. To separate DAPI and AF-488 emissions, we use band-pass 387 filters that allow passage of emitted light in the range of 435-485 nm (denoted as 460/50 nm) 388 and $510-550 \mathrm{~nm}$ (denoted as 530/40 nm), respectively.

390 The hierarchy of sorting gates we use are detailed in the methods below and in Fig. 2. After 391 nuclei are located in the plots described below and PMT settings are adjusted, sorting takes place 392 in real-time as the cytometry data are collected for each particle. Sorting parameters will need to 393 be carefully set to balance sample purity, sorting accuracy and speed (see your flow sorter 394 manual and $(43,46)$ for detailed description).

395 For users without prior experience in flow cytometry, we recommend partnering with an 396 established flow-core facility, in consultation with facility personnel. Specific instrument settings 397 will need to be determined empirically based on the experimental goals, sample type, and type of 398 flow sorter. The procedure and settings we used are for an InFlux (BD Biosciences) sorter and 399 have been described in detail in (32). Procedures for setting the Repli-seq sorting gates are 400 detailed below. 
1. Prepare the cytometer software to record forward light scatter (FSC), side light scatter (SSC), pulse height at 460/50 nm (DAPI fluorescence) and pulse height at 530/40 nm (AF-488 fluorescence). FSC is used to trigger and set the event threshold and both FSC and SSC are used in conjunction with 460/50 fluorescence to adjust the sorting gates and eliminate debris and broken nuclei. Steps 2-5 are done after starting the sample but before sorting begins.

2. Make dot plots of FSC versus SSC, FSC versus DAPI fluorescence, and SSC versus DAPI fluorescence (Fig. 2a) to locate and draw a gate encompassing 2C, 4C and 8C DNA content populations based on light scattering properties and DAPI fluorescence. Proper setting of these gates will reduce the amount of cellular debris in the target population and is described in detail in (32).

3. Many doublets, which are aggregates of two or more nuclei or cells (see Note 6), will be manually filtered out in step 3.4.9 but some will remain and be recorded as large, single particles as they cross the laser interrogation point. To differentiate them from single nuclei, create a dot plot of DAPI-W (460/50 nm pulse width) on the y axis by DAPI-A (460/50 nm pulse area) on the $\mathrm{x}$-axis. Locate and draw a gate encompassing the $2 \mathrm{C}, 4 \mathrm{C}$, of AF-488-H fluorescence (530/40 nm pulse height, log scale) versus DAPI-H fluorescence (460/50 nm height, linear scale) (Fig. 2c). This plot visualizes EdU labeled nuclei as an "arc" above the unlabeled, non-replicating G1 and G2 populations $(2 \mathrm{C}$ and 4C DNA contents, respectively) (Fig. 2c) (24, 26, 32). 
5. In the AF-488-H vs DAPI-H (530/40 nm vs 460/50 nm) dot plot (Step 4 above and Fig. 2c), draw a rectangular gate around each of the unlabeled G1 and G2 populations. Draw two rectangular gates in the labeled, S-phase arc directly above the G1 and G2 gates to capture early and late replicating nuclei, respectively. Then draw a rectangular gate midway between the early and late gates to capture replicating nuclei in the middle of S phase (Fig. 2c). Once the hierarchy for sorting has been established (see step 6 below), the gate drawn around G1 is used for sorting G1 nuclei. The gate drawn around G2 is used as a reference point for drawing the late S-phase gate but is not sorted, as G1 is sufficient to normalize for copy number and sequencability. DAPI dot plot is used as the first parent gate (PG1 in Fig. 2a) to remove debris and

6. With the gates drawn around the desired nuclei populations in each of the dot plots, establish a gating hierarchy such as is illustrated in Fig. 2a-c. In this example, the SSC vs broken nuclei. The events that lie outside PG1 are removed from further analysis and sorting. The events within PG1 are then analyzed using DAPI width vs DAPI area (460/50-W vs 460/50-A) to discriminate doublet and single nuclei (Fig 2b). Parent gate 2 (PG2) contains single nuclei, and these events are then analyzed in the AF-488-H (530/40 $\mathrm{nm})$ vs DAPI-H (460/50 nm) dot plot where the final early, middle, late and G1 sorting gates are established. for each plant species, type of experiment, and downstream application (see Note 7). Sort the final, gated nuclei into $2-\mathrm{mL}$ round-bottom tubes containing $500 \mu \mathrm{L}$ of $1 \times \mathrm{STE}$ (see Note 8). When large numbers of nuclei are needed, it may be necessary to use multiple 
tubes by replacing a tube that fills up during the sort with another tube. Alternatively, larger capacity tubes can be used to avoid handling multiple tubes (see Note 8). Multiple tubes from a single sort gate can be combined during downstream steps in the Repli-seq

\section{1}

452

\subsection{Reversing formaldehyde crosslinks and extracting genomic DNA} protocol.

453

454

455

456

457

458

459

460

461

462

463

464

465

466

467

468

469

1. After sorting, centrifuge the $2-\mathrm{mL}$ tubes at $850 \mathrm{xg}$ for $10 \mathrm{~min}$ at $4^{\circ} \mathrm{C}$ to pellet the nuclei.

2. Begin removing supernatant while carefully leaving $500 \mu \mathrm{L}$ of STE buffer over the invisible nuclei pellet. Discard the excess supernatant and resuspend the nuclei pellet by pipetting.

3. Prepare a solution of $1 \times \mathrm{STE}, 50 \mathrm{mM} \mathrm{Na} 2 \mathrm{EDTA}, 1 \%$ sodium lauroyl sarcosine and 200 $\mu \mathrm{g} / \mathrm{mL}$ proteinase $\mathrm{K}$ and add $500 \mu \mathrm{L}$ to each tube of sorted nuclei. Mix by pipetting up and down.

4. Incubate at $42^{\circ} \mathrm{C}$ for $1 \mathrm{~h}$ in the dark to remove proteins.

5. Incubate overnight at $65^{\circ} \mathrm{C}$ in the dark to reverse the formaldehyde crosslinks.

6. Divide each sample tube into two by placing $\sim 500 \mu \mathrm{L}$ into each of two phase lock tubes (Phase Lock Gel ${ }^{\text {TM}}$-Light Quantabio 5PRIME).

7. To purify genomic DNA, add 1 volume $(\sim 500 \mu \mathrm{L})$ of phenol:chloroform:isoamyl alcohol to the nucleic acid suspension in the phase lock tube and mix well by inversion. Centrifuge at $16,000 \mathrm{xg}$ for $5 \mathrm{~min}$ at $22^{\circ} \mathrm{C}$. Transfer the upper aqueous phase containing the DNA into a new phase lock tube.

8. To remove the residual phenol, add one volume $(\sim 500 \mu \mathrm{L})$ of chloroform:isoamyl alcohol to the nucleic acid suspension and mix well by inversion. Centrifuge at $16,000 \times \mathbf{g}$ for 5 $\min$ at $22^{\circ} \mathrm{C}$. 
9. Transfer the upper aqueous phase containing the DNA into a new phase lock tube and repeat step 3.6.8 one more time. Transfer the final aqueous phase into a clean 2-mL round-bottom centrifuge tube. mixture. This will aid in the precipitation of small amounts of DNA and visualization of the DNA pellet. $\mathrm{NaOAc})$ and $0.3 \mathrm{~mL}$ (0.6 volumes) cold isopropanol. Invert the tube 15-20 times and incubate at $-20^{\circ} \mathrm{C}$ for $1-4 \mathrm{hrs}$.

12. Pellet the DNA at $20,000 \mathrm{x} \mathbf{g}$ for $30 \mathrm{~min}$ at room temperature and discard the supernatant.

13. Wash the blue pellet twice with $1 \mathrm{~mL}$ of $70 \% \mathrm{EtOH}$. Gently tap the side of the tube to dislodge the pellet after each wash. Centrifuge at 20,000 $\mathrm{xg}$ for $15 \mathrm{~min}$ at room temperature and discard the supernatant. vacuum concentrator with heating. concentration using a Qubit dsDNA High Sensitivity DNA assay kit.

491 The shearing and immunoprecipitation steps detailed below are an adaptation of the ChIP-chip 492 protocol described in (42). Specific volumes and settings are based on use of a Covaris S220 
493 Ultrasonicator. To ensure that the AF-488 immunoprecipitation is specific, we recommend that 494 the binding reaction take place in a $1 \times$ ChIP dilution buffer.

495 1. Adjust the final volume of each DNA sample from step 3.6.16 to $120 \mu \mathrm{L}$ in ChIP dilution buffer, ensuring a final concentration of $1 \times$. Transfer the entire volume into a 130- $\mu$ L Covaris microTUBE AFA Fiber Pre-Slit Snap-Cap tube.

2. Shear the DNA to an appropriate size. In our experiments with maize, we sheared to an average size of $250 \mathrm{bp}$ for 100-bp paired-end libraries. To achieve this, we used the following settings on the Covaris S220: Peak Incident Power 175; Duty cycle 10\%, Cycles/burst 200; Time 180 sec. For longer reads (e.g. 150-bp paired-end reads), larger fragments may be desirable, for which the appropriate Covaris settings will need to be empirically determined.

3. After shearing, adjust the sample to a total volume $500 \mu \mathrm{L}$ using $1 \times$ ChIP dilution buffer

4. Before use, pre-wash an aliquot of Dynabeads ${ }^{\circledR}$ Protein $G$ magnetic beads. Mix the magnetic beads until they are uniformly suspended. Remove $20 \mu \mathrm{L}$ of the bead slurry and place in a clean 1.5-mL low binding tube. To wash the beads, add $0.5 \mathrm{~mL}$ of ChIP dilution buffer and mix by inversion or vortexing until the beads are in suspension. Capture the beads for 2 min on a magnetic rack until the supernatant is clear and discard the supernatant. Repeat the wash step two more times. After the last wash, add $20 \mu \mathrm{L}$ of ChIP dilution buffer to the beads and resuspend by pipetting up and down.

5. Pre-clear the sheared genomic DNA solution by adding $20 \mu \mathrm{L}$ of pre-washed Dynabeads ${ }^{\circledR}$ Protein $\mathrm{G}$ magnetic beads per $500-\mu \mathrm{L}$ sample in a clean $1.5-\mathrm{mL}$ low 
binding tube. This will aid in removing any molecules that may bind nonspecifically to the Protein $\mathrm{G}$ beads and improve the signal to noise ratio of the antibody immunoprecipitation. Incubate the tubes in the dark at $4^{\circ} \mathrm{C}$ for $1 \mathrm{~h}$ with gentle mixing. We use a Nutator ${ }^{\mathrm{TM}}$ mixer with gentle, end-over-end mixing. Capture the beads for 2 min on a magnetic stand and transfer the supernatant containing the pre-cleared DNA to a new $1.5-\mathrm{mL}$ tube and discard the beads.

6. To immunoprecipitate the EdU-labeled DNA, add 1:200 dilution of anti-AF488 (rabbit IgG, A-11094, Molecular Probes) antibody to each pre-cleared DNA sample and incubate overnight at $4^{\circ} \mathrm{C}$ in the dark with gentle end-over-end mixing.

7. Pre-wash $25 \mu \mathrm{L}$ of Dynabeads ${ }^{\circledR}$ Protein $\mathrm{G}$ magnetic bead slurry three times in 0.5 $\mathrm{mL}$ of ChIP dilution buffer. After the last wash, resuspend the beads in $50 \mu \mathrm{L}$ of ChIP dilution buffer.

8. To precipitate the DNA-antibody complex, add $50 \mu \mathrm{L}$ of the pre-washed beads to the tube containing $500 \mu \mathrm{L}$ of DNA-antibody complex solution and incubate the tubes at

10. Capture the beads containing the DNA-antibody complex for 2 min on a magnetic stand and discard the supernatant. $4^{\circ} \mathrm{C}$ for $2 \mathrm{~h}$ in the dark with gentle end-over-end mixing.

9. During this incubation period, prepare the ChIP wash buffers at room temperature and place on ice. Prepare fresh elution buffer and keep at room temperature to avoid SDS precipitation.

1. To remove non-specific binding, wash the beads containing the DNA-antibody complex in a series of four wash buffers in the following order: Low-salt wash, Highsalt wash, $\mathrm{LiCl}$ wash and TE wash. Wash the beads three times in each buffer, using 1 
$\mathrm{mL}$ of buffer in each washing step. The first wash is a quick wash. For the two subsequent washes, incubate 5 min on an end-over-end mixer, capture the beads on a magnetic stand and discard the supernatant. Do all steps at $4^{\circ} \mathrm{C}$.

542

12. After the final wash, carefully remove as much TE as possible.

13. Elute the immune complexes from the beads with freshly made elution buffer at $65^{\circ} \mathrm{C}$. Add $200 \mu \mathrm{L}$ of elution buffer to the washed beads and mix gently by pipetting up and down. Incubate at $65^{\circ} \mathrm{C}$ for $15 \mathrm{~min}$ and gently mix by inverting the samples five times every $3 \mathrm{~min}$. Capture the beads for $2 \mathrm{~min}$ and transfer the supernatant to a new tube.

14. Repeat step 3.7.13 one more time. Combine the two eluates for a total volume of 400 $\mu \mathrm{L}$.

15. To digest the antibody and any proteins in the solution, add $16 \mu \mathrm{L}$ of $1 \mathrm{M}$ Tris, $\mathrm{pH}$ $6.5,16 \mu \mathrm{L}$ of $5 \mathrm{M} \mathrm{NaCl}, 8 \mu \mathrm{L}$ of $0.5 \mathrm{M} \mathrm{Na}_{2}$ EDTA and $1 \mu \mathrm{L}$ of proteinase $\mathrm{K}(23-27$ $\mathrm{mg} / \mathrm{mL})$. Mix well and incubate at $45^{\circ} \mathrm{C}$ for $1 \mathrm{~h}$.

\section{$553 \quad 3.8$ Purifying immunoprecipitated DNA}

554 The immunoprecipitated (IP), EdU-labeled DNA fragments should be purified before making 555 DNA sequencing libraries. Because the DNA has been sheared to a small size, use a DNA 556 purification kit designed for purification of fragmented DNAs. Prepare all kit buffers per the 557 manufacturer's instructions before proceeding with the DNA purification steps. We have used 558 the QIAquick PCR Purification Kit, and have detailed our modifications to the procedure below. 559 All centrifugations are at $17,900 \mathrm{xg}$, at room temperature.

1. Place the DNA sample $(\sim 440 \mu \mathrm{L})$ into a 5 -mL round-bottom tube. 
2. Add 5 volumes $(2200 \mu \mathrm{L})$ of Qiagen Buffer PB to the DNA sample and mix well by pipetting.

3. Add $40 \mu \mathrm{L}$ of $3 \mathrm{M} \mathrm{NaOAc}, \mathrm{pH} 5.2$, to each tube to adjust the $\mathrm{pH}$ to that required for column binding. Mix well.

565 4. Place a QIAquick spin column in a 2-mL collection tube.

5665 . Apply $1000 \mu \mathrm{L}$ of the sample to the center of the QIAquick column to bind DNA.

567 Centrifuge for $60 \mathrm{sec}$

568 6. Discard the flow through. Place the QIAquick column back into the same tube.

569 7. Repeat the DNA binding (steps 5-6) for a total of 4 times with IP DNA or until all the $570 \quad$ sample has passed through the column.

5718 8. Wash the column by adding $0.75 \mathrm{~mL}$ Qiagen Buffer PE and centrifuge for $60 \mathrm{sec}$.

572 9. Discard the flow through and place the QIAquick column back into the same tube.

573 10. Centrifuge for an additional $1 \mathrm{~min}$ to remove any residual liquid.

574 11. Place the QIAquick column in a clean 1.5-mL low binding tube and elute the DNA from

575 the column by adding $35 \mu \mathrm{L}$ of Qiagen Buffer EB to the center of the QIAquick column.

$576 \quad$ Let the column stand for $2 \mathrm{~min}$ and then centrifuge for $60 \mathrm{sec}$.

577 12. Measure the concentration of eluted DNA using $5 \mu \mathrm{L}$ elute in the Qubit dsDNA High $578 \quad$ Sensitivity DNA assay.

579 13. Store the purified IP DNA at $-80^{\circ} \mathrm{C}$ until library preparation.

\section{$580 \quad 3.9$ Preparing the DNA sequencing library}

581 The following protocol is derived from the instructions for the NEBNext Ultra II DNA library

582 preparation kit. 
1. Use $500 \mathrm{pg}$ to $1 \mu \mathrm{g}$ of purified DNA per library, adjusting to a final volume of $50 \mu \mathrm{L}$ with nuclease-free water in a sterile, nuclease-free $200-\mu \mathrm{L}$ tube. and $7 \mu \mathrm{L}$ of End Prep Reaction Buffer. Mix thoroughly by pipetting, briefly centrifuge to bring down any droplets on the side of the tube, and incubate for $30 \mathrm{~min}$ at $20^{\circ} \mathrm{C}$. Denature the enzymes for $30 \mathrm{~min}$ at $65^{\circ} \mathrm{C}$, and hold at $4^{\circ} \mathrm{C}$.

3. Ligate the NEBNext adapter for Illumina to the end-repaired DNA fragments. Before ligation, dilute the adapter according to the NEBNext kit directions, based on the amount of starting DNA. Set up the ligation reaction by adding $30 \mu \mathrm{L}$ of Ultra II Ligation Master heated lid off.

4. Cleave open the hairpin NEBNext adapter for Illumina by adding $3 \mu \mathrm{L}$ of USER Enzyme directly to the ligation reaction and mix by pipetting up and down. Quickly centrifuge to bring down droplets from the side of the tube, and incubate at $37^{\circ} \mathrm{C}$ for $15 \mathrm{~min}$ in a thermocycler with the lid set to $\geq 47^{\circ} \mathrm{C}$. USER Enzyme is only necessary if NEBNext adapters are used. Samples can be stored at $-20^{\circ} \mathrm{C}$.

5. Size select and clean up the adapter ligated DNA fragments. As per the NEBNext Ultra II DNA library kit instructions, if the amount of starting DNA is greater than $50 \mathrm{ng}$, size selection should be carried out with the SPRIselect beads. If the starting sample is $50 \mathrm{ng}$ or less, then to maintain the complexity of the library only a clean-up of the sample is 
volume of SPRIselect beads, recognizing that the volume will depend on the desired fragment size for the library. Magnetic beads selectively bind to nucleic acid fragments of different sizes based on a volume ratio of the bead suspension to nucleic acid solution. It is therefore important that the bead suspension is well mixed before use and is pipetted slowly and accurately (see Note 9).

6. For size selection, add the recommended volume of resuspended SPRIselect beads to the ligation reaction and mix well by pipetting up and down. Incubate the samples for 5 min at room temperature. Briefly centrifuge to bring down droplets from the side of the tube, and place the tubes on a magnetic stand to separate the beads from the supernatant. Let stand for 5 min or until the beads and solution have separated. Carefully remove the supernatant with a pipet and transfer to a new tube. The beads contain large, unwanted DNA fragments and can be discarded.

\section{Add the second recommended volume of well-mixed beads to the supernatant and mix} well. Let stand for $5 \mathrm{~min}$ at room temperature. Briefly centrifuge to bring down droplets from the side of the tube, and place the tube on a magnetic stand for $5 \mathrm{~min}$ or until the beads and solution are separated. This time, discard the supernatant and keep the beads which have the desired sized DNA fragments.

8. Wash the beads $2 \times$ with freshly prepared $80 \% \mathrm{EtOH}$, keeping each wash on the beads for $30 \mathrm{sec}$. After the second wash, remove the EtOH. Briefly spin down the samples to bring any residual EtOH to the bottom of the tube and place back on the magnetic rack. After 
9. Air dry the beads for up to 5 min with the lid open. Do not over dry the beads or DNA recovery may be reduced. Remove the tube from the magnet and elute the DNA from the beads by mixing with $17 \mu \mathrm{L}$ of $0.1 \times \mathrm{TE}$. Let stand for $2 \mathrm{~min}$ at room temperature. Briefly centrifuge to bring down droplets from the side of the tube, and place on a magnetic rack for $\sim 5$ min until the supernatant is clear.

10. Transfer $15 \mu \mathrm{L}$ of supernatant to a new $200-\mu \mathrm{L}$ PCR tube and proceed to PCR

635 amplification of the library.

11. For PCR amplification, add $25 \mu \mathrm{L}$ of Ultra II Q5 master mix and $10 \mu \mathrm{L}$ of a unique dual index set (see Note 10) directly to the size-selected/cleaned, adapter-ligated DNA fragments for a total volume of $50 \mu \mathrm{L}$. Mix thoroughly by pipetting and briefly centrifuge to bring down droplets from the side of the tube. Depending on the initial amount and nature of the DNA starting material, the number of PCR cycles will need to be determined empirically.

12. Follow the recommendations in the NEBNext Ultra II kit instructions to determine the number of amplification cycles needed. Amplify the library with the following conditions:

644

\begin{tabular}{|c|c|c|c|}
\hline Cycle Step & Temperature & Time & $\begin{array}{c}\text { Number of } \\
\text { Cycles }\end{array}$ \\
\hline Initial denaturation & $98^{\circ} \mathrm{C}$ & $30 \mathrm{sec}$ & 1 \\
\hline Denaturation & $98^{\circ} \mathrm{C}$ & $10 \mathrm{sec}$ & \multirow{2}{*}{$3-15$} \\
\hline $\begin{array}{l}\text { Annealing and } \\
\text { extension }\end{array}$ & $65^{\circ} \mathrm{C}$ & $75 \mathrm{sec}$ & \\
\hline Final extension & $65^{\circ} \mathrm{C}$ & $5 \mathrm{~min}$ & 1 \\
\hline Hold & $4^{\circ} \mathrm{C}$ & Hold & - \\
\hline
\end{tabular}


13. Clean up the reaction using SPRISelect beads after PCR amplification. Follow the steps as described in section 3.9.6-3.9.10 for size selection/clean up after the adapter ligation step, except use a bead to sample volume ratio of 0.9 to clean up the PCR reaction. This ratio will remove excess primers and salts from the PCR reaction. Use $33 \mu \mathrm{L}$ of nucleasetube. (see Note 11).

14. If pooling libraries for sequencing on the same flow cell, normalize the library concentrations. First, analyze the quality and the size distribution of the library fragments 
669 Repli-seq reads are quality controlled, trimmed and mapped to the genome using BWA-MEM

670 (51). Alignments are filtered to remove improperly paired reads, duplicates, and alignments to

671 which BWA-MEM assigns low mapping quality (MAPQ) scores. MAPQ is estimated by BWA-

672 MEM as a metric reflecting the probability that a read is aligned to the wrong location in the

673 genome (52). The maize genome has a large proportion of repetitive DNA, making it imperative

674 to focus on unique alignments (see Note 15). After filtering, the result is properly paired,

675 uniquely mapped reads as input for the Repliscan software (section 3.11) for further processing

676 and final classification of replication time at each locus.

677 All quality control, alignment and alignment filtering steps detailed here can be performed using

678 command line scripts (as described in refs $(51,53-56)$ ), however we will focus on an analysis

679 pipeline (Table 1) that uses user-friendly, point-and-click applications (apps) implemented in the

680 CyVerse Discovery Environment (DE; (57)). For each app, the needed input and resulting output

681 files are listed in Table 2. The parameters/options to use are described in the steps below and

682 subsequent notes as well as example screenshots shown in Figs. S1-S10. A general introduction

683 to navigating and using apps in the DE can be found at

684 https://cyverse.atlassian.net/wiki/spaces/DEmanual/overview (also see Note 16).

685 1. Quality Control. To improve alignment rates and reduce errors, use the Trim-galore-

686 0.4.1 app with default parameters to remove low quality and adapter sequences from the 687 sequencing read files (see Note 17 and Fig. S1).

688 2. Alignment. Align the quality-controlled reads (preferably paired-end; see section 3.9.16)

689 to the desired reference genome using the BWA_mem_0.7.15 app with default

690 parameters (Fig. S2). 
3. Post-Alignment Filtering. Due to the repetitive nature of the maize genome, only unique alignments whose orientations made them proper pairs should be included in the processed alignment files for downstream analysis. Perform the following filtering for each alignment file. Use the SAMtools view 1.9 app to filter out reads that are unmapped and in non-primary alignments (SAM flag: 0x104 checkbox), reads that are not properly paired (SAM flag: 0x2 checkbox), and reads that are supplementary alignments (SAM flag: $0 x 800$ checkbox). The SAMtools view 1.9 app can also filter out reads that are not mapped confidently to a single location, as assessed by the MAPQ assigned by BWAMEM. The MAPQ for alignments in a dataset can be assessed by plotting the MAPQ distribution and determining a proper threshold (see Note 15 and Fig. S3).

4. Duplicate Read Filtering. Use the Picard-MarkDuplicates-2.7.1 app to remove duplicate reads (see Note 18 and Fig. S4).

5. Basic Alignment Statistics. Use the SAMtools 1.7 Flagstat app to generate alignment statistics (Fig. S5). If desired, this app can be run at multiple steps in the alignment filtering process with any alignment .bam or .sam file (e.g. see Table 3).

6. Biological Replicate Agreement. Use the deeptools_3.2.0 app, which implements the DeepTools multiBamSummary tool to assess the agreement between individual biological replicates using Pearson's correlation coefficient (see Note 19 and Fig. S6). Correlation coefficient values greater than 0.8 are considered sufficient, but we routinely see values of 0.9 or higher.

7. Optional Random Downsampling. Repli-seq results are robust at various sequencing depths (29). However, when different RT profiles are to be quantitatively compared it may be desirable to randomly downsample some files of filtered, uniquely aligned reads 
so that the RT profiles to be compared are all derived from data of comparable depth (see

Note 20).

$717 \quad 3.11$ Analyzing and classifying replication time using the Repliscan application

718 Repliscan is custom-built software for analysis of Repli-seq data produced by label-incorporation

719 (e.g. EdU or BrdU). Command line scripts and parameters are described in detail in Zynda et al.

720 (29). Here, we will focus on the point-and-click Repliscan application available in the CyVerse

721 DE. A Repliscan app manual and test data instructions can be found at

722 https://cyverse.atlassian.net/wiki/spaces/DEapps/pages/673841153/Repliscan. The test data

723 hosted on CyVerse (/iplant/home/shared/iplantcollaborative/example_data/repliscan) is aligned

724 to B73 Refgen_v4, filtered and randomly downsampled from the original dataset (NCBI SRA

725 accession PRJNA327875). The dataset does not include individual biological replicate data in an

726 effort to reduce file size. Repliscan default parameters (Fig. S7) will most likely produce good

727 results, provided the dataset has sufficient sequencing depth (see Note 14). However, we also

728 describe some of the most common modifications to a few key parameters, and include

729 recommended parameters for running the test data in Fig. S8.

730 1. Input Configuration. Create an input configuration input.txt file to assign files to the 731 appropriate sample names (Fig. S9a). Individual biological replicate .bam files for the G1

732 (2C) reference control and each S-phase sample are used (after filtering; see sections

$733 \quad 3.10 .3-3.10 .4$ ) as input files for the Repliscan 0.1.0 app. Individual files should be

734 delimited by a tab. The configuration input.txt and .bam files should be located together

735 in a CyVerse directory. The path to the desired reference genome $f a$ file is also required

736 as input (see Table 2 and Fig. S9b) 
2. Analysis Bin Size. Read densities are calculated in a static bin size across the genome. The default analysis bin size (1000 bp) is useful to highlight transitions between replication times, but may be too small for low-coverage data. Adjust the analysis bin size in base pairs parameter according to your experimental needs (see Note 21). reads from individual biological replicates from the corresponding bins of each replicate. If you have extremely high read depth (e.g. (27)), the most robust aggregation method is to select median (29), which calculates the median of the scaled read densities from different biological replicates in a given bin. read density in the G1 control may be removed from the analysis using the remove parameter and the dependent parameter percentile cutoff. The default setting is remove norm and the default for percentile cutoff is the percentile range 2.5-97.5. These settings can be adjusted according to experimental needs (see Note 22).

5. Sequence Depth Scaling. Repliscan normalizes each aggregated sample dataset using sequence depth scaling to $1 \times$ reads per genomic content (RPGC; (58)). How the scaling is handled depends on which aggregate method (section 3.11.3) is chosen (see Note 23).

6. Normalization to a Non-replicating Control. The G1 control data are used to normalize for sequencing biases, read mappability variation, and differences in copy number. The scaled read density in a given bin from each of the S-phase samples are divided by the scaled read density from the corresponding bin in the non-replicating G1 control. The result is a ratio of the S-phase sample to the G1 control value in each bin, and represents an estimate of the intensity of replication activity in that bin ("replication signal") for that 
portion of S-phase. If G1 control data are not available (e.g., in some cases where preexisting data are being analyzed), data from an unsorted control sample, or pooled data from the entire S-phase can also be used. However, these procedures are less than optimal (see Note 24).

764 7. Haar Wavelet Smoothing. To reduce statistical noise, the ratio data for each S-phase sample is smoothed using a Haar wavelet transform (59), which accomplishes smoothing without spreading peak boundaries or lowering resolution. The default for the Haar smoothing level parameter is level 3 as it effectively removes low-amplitude noise while preserving the overall structure of the profiles.

8. RT Class Segmentation. The final step in a Repliscan analysis is to incorporate the replication signal for each S-phase portion in each bin and make a qualitative call of the predominant replication time class (RT class) in that bin. The parameters, scope,

773 the algorithm used for the RT class segmentation. We recommend using the default settings for all of these parameters (see parameter settings in Fig. S7), but a more inchromosome (parameter scope - chromosome). The largest replication signal value is always classified as replicating, and the algorithm allows multiple time classifications (e.g. middle and late) when another signal is within $50 \%$ of the highest value (parameter classifier-proportion). If data from three S-phase slices (early, middle and late) are used 
as input, the resulting RT class options are: not-segmented, early, middle, and late, as well as the multiple time classes: early-mid, mid-late, early-mid-late and early-late.

9. Repliscan Output. Repliscan outputs .bedGraph files from each step of the pipeline and a final color-coded RT class segmentation .gff3 file (Table 2 and Fig. 4d). The files can be downloaded from CyVerse and loaded into a genome browser, such as IGV (Fig. 4). Additional output files are created by selecting the plot check box. These include a plot showing the distribution of G1 reads per bin (G1_coverage_cut.png; Fig. S10a) from the remove parameter (section 3.11 .4 ) and a $\log$.out file that contains all the parameter settings used and the automatically tuned segmentation thresholds (Fig. S10b).

\section{Notes}

793

1. EdU concentration and pulse length. The optimal EdU concentration and pulse length need to be determined and may vary depending on meristem type, plant species, or cell culture. For determining the optimal EdU pulse length, knowing the approximate duration of S phase and the approximate length of time it takes the EdU precursor to enter the experimental material is useful. In maize root tips, S-phase duration is estimated to be 2.7-3.9 hours (24) and a 20-min pulse length, which represents about $\sim 10 \%$ of the length of S phase, gives an excellent separation of replicating nuclei from the unlabeled G1 and G2 populations as compared to 5- and 10-min pulse lengths (Fig. 3). A flow cytometric analysis of an EdU pulse labeling time course illustrates the differentiation of labeled and unlabeled nuclei at 5, 10, and $20 \mathrm{~min}$. It is clear in maize root tips that even at $5 \mathrm{~min}$, EdU is being incorporated into newly synthesized DNA (Fig. 3) as an "arc" of labeled S-phase nuclei is starting to form above the unlabeled G1 and G2 populations. At 10 and 20 min 
the EdU-labeled S-phase nuclei are further differentiated from the unlabeled G1 and G2 populations. It is also noteworthy that labeling with EdU allows for clean separation between labeled and unlabeled nuclei with the same DNA content (Fig. 2c, d), producing a sort purity that is nearly impossible if sorting on DNA content alone as is required when labeling with BrdU. EdU has been reported to impair cell cycle progression in several mammalian cell lines where cytotoxic effects were observed in a cell type, dose, and time dependent manner, with most perturbations seen in subsequent cell cycles after EdU incorporation and not in the initial cell cycle in which EdU is incorporated (60-63). When using EdU to label replicating DNA, it is important to analyze the experimental material for any cell cycle perturbations that may result after EdU treatment, especially in studies of long-term EdU exposure (61). In Repli-seq studies, the cells that take up EdU label are immediately harvested after the short labeling period, therefore reducing any negative long-term implications that EdU incorporation may have on the cell cycle and DNA

2. Root tip length for dissection. In maize, distinct regions of development have been identified along the root. The root, starting with the apex, contains meristematic cells, which are followed by transition and elongation zones $(\mathbf{6 4}, \mathbf{6 5})$. Using flow cytometry and a series of 1-mm dissected segments up the maize root, we identified that relative to other 1-mm segments, the first $1 \mathrm{~mm}$ from the apex contains the highest percentage of actively dividing cells (30). In root tips of different species, a strategy similar to Bass 2014 (30) for identifying the region of highest mitotic activity can be employed. your needs. Over fixation can make it difficult to reverse crosslinks prior to the 
immunoprecipitation of EdU-labeled DNA. To minimize the amount of time that roots are in the fixative, label maize seedlings in small batches (e.g. 100-200 seedlings) and have two or more people assist in root dissection. The labeled, dissected batches of root tips may then be pooled during nuclei isolation.

833 to damage or nick nucleic acids (67-69). In a titration study, we found that we could

4. Click $i T \circledR$ Reaction Cocktail. $\mathrm{CuSO}_{4}$ catalyzes the click reaction (66), and $\mathrm{Cu}^{+2}$ is known reduce the molarity of $\mathrm{CuSO}_{4}$ to half that recommended by the Click iT kit instructions without negatively affecting the AF-488 coupling reaction. To minimize the nuclei from sticking together and forming clumps, we added Triton X-100 to a final concentration of $0.1 \%$ to the Click iT ${ }^{\circledast}$ Reaction Cocktail.

5. Alternatives to DAPI stain for total DNA. For flow sorting, fluorescent nucleic acid stains should bind stoichiometrically so as to accurately reflect the amount of DNA present in each nucleus. In our sorting experiments for Repli-seq, we use DAPI to stain total DNA because it has low spectral overlap with AF-488, binds specifically to double-stranded DNA and gives high quality G1 histograms with small coefficients of variation (for a presentation of the accuracy of DNA content measurement see $(32,48,70)$ and references within). In this protocol, nuclei are stained and sorted but for applications using whole cells, the cells would first require permeabilization as the plasma membrane is impermeable to DAPI. Other examples of fluorescent stains used for nucleic acid analysis during flow sorting include propidium iodide, DRAQ5 and 7AAD, Hoechst 33342 and 33258, ethidium bromide, acridine orange, thiazole orange (TO and YO-PRO1), chromomycin A3, and Vybrant DyeCycle Ruby, a proprietary DNA staining dye. Points to consider when choosing a dye include whether analysis is for plant or animal 
cells/nuclei (70), cell permeability, whether the stain binds to RNA and/or DNA (71), use of living cells versus fixed cells (48), effect of $\mathrm{pH}$ on fluorescence, whether the dye is quenched in the presence of other fluorophores, the dye's absorption and emission, the laser configuration of the sorter (45), and spectral overlap with other fluorescent dyes during multi-parametric analysis. When staining live cells, long exposure to certain dyes can induce DNA damage response, which may perturb the cell cycle (48). and recorded as a single, larger particle as they cross the laser interrogation point (49). Based on DNA content, a doublet of two G1 nuclei, for example, would be sorted as a single G2 nucleus. Taking advantage of the flow cytometry parameters of pulse height, width, and area, one can reduce the number of doublets from the analyses and sorting (See Figure 3 of (49) for an example of flow cytometric doublet discrimination). Two G1 nuclei stuck together are larger than a single G2 nucleus and will typically take longer in units of time to pass through the beam than a single G2 nucleus. In a plot of height vs. width, the area under the curve for a G1 doublet vs. a G2 singlet will be different. These measurements, however, can vary based on the orientation of the doublet as it passes through the beam (46) and may vary among cell types (71). The gate we used to minimize doublets employs the parameters of width and area (Fig. 2b). A further consideration when detecting doublets, is the beam geometry of the sorter. Instruments can have either more elliptical or oval beam geometries, and this affects the size of the beam spot (46). Sorters with smaller beam spots are more likely to analyze a doublet as a single event (72). Therefore, being familiar with the sorter's optical dimensions and 
establishing a gating strategy using height, width and area will be critical for removing doublets, for correct interpretation of the cell cycle and for sorting pure populations.

7. Numbers of nuclei to sort for Repli-seq. Genome size, immunoprecipitation efficiency, and amount of starting DNA required for library preparation are all important considerations when determining the number of nuclei to be sorted for each experiment. In our initial Repli-seq experiments in maize, with a genome size of $2.3 \mathrm{Gbp}$ (73), we sorted 500,000 to 1,000,000 nuclei for each S-phase and G1 fraction to produce sufficient amounts of DNA for downstream analysis (26). DNA yields from these numbers of nuclei typically ranged from 1200-2200 $\mu$ g. After shearing and immunoprecipitation, typical yields of $18-40 \mathrm{ng}$ of DNA (1-2\% of total input) were available for library preparation. Current library kits require very little starting material and $0.5 \mathrm{ng}$ DNA is a common starting amount on the low end of the recommended range. It is now feasible to sort fewer maize nuclei than were sorted in the initial Repli-seq experiments and yield sufficient amounts of DNA for making Repli-seq libraries. However, a good library will retain as much of the starting material's original complexity as possible and larger amounts of starting material will require less PCR amplification (74). sorted nuclei and handling after sorting can vary from that used for Repli-seq analysis. For other applications, like microscopy $(22,30)$ or ChIP-seq $(26)$, sort nuclei into a $2 \times$ concentration of CLB without 2-mercaptoethanol or 2× Extraction Buffer 2 (42), respectively. After sorting, ensure that the sample is diluted to a $1 \times$ concentration by adding additional $1 \times$ STE. For microscopy, nuclei can be stored in $1 \times$ CLB without 2 - 
(22). Also, a variety of sorting platforms and tubes are available depending on the sort needs and the flow cytometer being used. Accommodate for any differences in the 2-mL sort tube described here.

899

9. Working with SPRIselect beads. SPRIselect magnetic beads or similar products selectively bind to nucleic acid fragments of different sizes based on a volume ratio of the

901 supplied bead suspension to nucleic acid solution. It is imperative that the beads are well mixed just before beginning any bead-based selection or clean up so that all the samples receive the same volume-to-volume ratio of beads. Because the beads settle, it is also good to quickly mix them before every addition to a new tube to ensure that all tubes receive the same ratio of beads. Any changes to volume ratios, especially when working with small volumes, impacts the size of DNA fragments that are selected and can cause variability among library preparations. Therefore, the volume of beads should be measured slowly and accurately and any excess drops of beads on the pipet tip should be

10. Indexes for sequencing. Illumina sequencing platforms have steadily increased output and removed (75). their newest platform, NovaSeq 6000 generates up to 20 billion reads per run. To exploit these high output platforms, labs routinely pool hundreds of libraries as a cost savings and experiment scalability benefit. Pooled libraries are then de-multiplexed in silico. assignment of sequencing reads and incorrect assumptions in downstream analysis (7678). Index hopping is most commonly seen when using combinatorial indexes or using Illumina platforms that use patterned flow cells (ExAmp cluster generation) versus nonpatterned flow cells (bridge-amplification) (76-78). To mitigate index hopping, it is best 
to use unique dual indexes (UDI) which are distinct, non-redundant index sequences for each of the 17 and i5 indexes. In this scenario, if purchased in a 96-well format, there are no repeated indexes across columns or rows such that every combination of i7 and i5 indexes is unique. Combinatorial indexes, on the other hand, when purchased in a 96-well format, typically have unique i7 indexes across 12 columns and unique i5 indexes across 8 rows. In this format, there are redundant uses of both the $\mathrm{i} 7$ and $\mathrm{i} 5$ primers. For example, in wells A1-8 of a 96 well plate, the same i7 index will be paired with 8 different i5 indexes (See Figure 1 of (79)). In addition to using UDIs, other best practices to reduce index hopping when pooling multiple libraries are removing free adapters and buffer or water. A volume of $33 \mu \mathrm{L}$ is suggested in the NEBNext kit protocol. This volume works well for most samples, but slightly larger or smaller volumes may also be concentrated by vacuum concentration, an additional bead selection step, or ethanol used. Occasionally, the eluted DNA may be too dilute to meet the minimum DNA molarity requirements of the sequencing facility. In this case, the sample will need to be precipitation. We prefer the simpler procedure of concentrating sample(s) under vacuum. This requires carrying out the elution step with water rather than with a buffer solution to avoid concentrating buffer salts along with the DNA. If the samples are eluted in a buffer, vacuum concentration will also concentrate the buffer salts, which may inhibit the 
sequencing reactions. In this case, bead selection or ethanol precipitation may be the better choice for concentrating the DNA. However, some DNA will be lost during the extra steps required by these methods. convert mass concentration to molar concentration as recommended by Illumina (80), use the following formula: $\frac{(\text { Mass concentration in } \mathrm{ng} / \mu \mathrm{L})}{(660 \mathrm{~g} / \mathrm{mol})(\text { average fragment size in bp })} \times 10^{6}=$ concentration in $\mathrm{nM}$

The mass concentration of the library is determined by Qubit fluorometry; $660 \mathrm{~g} / \mathrm{mol}$ is the average molecular weight of a base pair of DNA; and the average fragment size of the library is determined using the Bioanalyzer. The fragment size profile should have an approximately normal distribution at a single peak. The size distribution is important because smaller library fragments cluster more efficiently than larger fragments on the profile of the library is too broad, has a double peak, or is heavily skewed, consideration should be given to another round of bead-based size selection or to remaking the library. Quantitative PCR (qPCR) is an alternative option for determining concentrations of while manual calculation merely determines the concentration of all DNA present. Determining concentration by qPCR is especially recommended when making PCR-free libraries, where enrichment of properly ligated DNAs has not taken place (81). Some 
library kits, like NexteraXT, avoid qPCR and manual calculations altogether, and offer bead based normalization steps. When working within the quantification limits of both Qubit and the Bioanalyzer assay kits, we routinely obtain successful, balanced sequencing runs by determining concentrations manually. fragments and cluster more efficiently on the sequencing flow cell. Because of clustering discrepancies, it is not only important to restrict the range of fragment size within a given library but also that pooled libraries have similar size distributions. Pooling libraries with a large range of insert sizes makes cluster prediction inaccurate. Therefore, it is and cluster generation see (81). for a Repli-seq experiment depends on the genome size, the number of S-phase portions examined and the desired bin size. In maize, with a $2.3 \mathrm{Gbp}$ genome (73) and a 3-kb bin size, we have used as few as 65.7 million uniquely mapped reads $(\sim 2.8 \times$ reads per genomic content) for each S-phase sample (early, middle and late) after combining biological replicates. To determine the total number of sequencing reads to obtain, consider the expected number of reads that will be lost to alignment filtering steps (section 3.10.3-3.10.4; see Table 3). More reads are always desirable, if the budget allows. We chose to pool three biological replicates of G1 nuclei to generate a single G1 control library, but multiple biological replicate libraries for G1 can be sequenced. Additionally, we recommend sequencing the G1 control library (or libraries) to a similar 
depth as the S-phase libraries. Zynda et al. 2017 (29) demonstrated that Repli-seq data produce stable results over a wide range of sequencing depths. This was done by carrying out a random downsampling analysis with data from Arabidopsis (0.13 Gbp genome; (82)). Using Repliscan software default parameters (section 3.11) and a 1-kb bin size, results were quite stable when data were downsampled from an average of $\sim 40$ million reads $(\sim 30 \times$ reads per genomic content) to $\sim 3.2$ million reads $(\sim 2.4 \times$ reads per genomic content) per library. When this depth cannot be achieved, a larger Repliscan analysis bin size can be considered (see Note 21).

15. Plotting and Filtering by Mapping Quality. The mapping quality (MAPQ) range is estimated by BWA-MEM as a metric of the probability that a read is aligned to the wrong location in the genome (52). Each alignment tool may have a somewhat different method of calculating MAPQ, so the results are not always consistent across tools (83). In BWAMEM, MAPQ ranges from 0-60 with zero representing a read that has received a random alignment location out of many possible locations and 60 representing a high confidence unique alignment. In our experience and that of others (e.g. (83)), the vast majority of alignments receive a MAPQ of close to 0 or 60 , with a small fraction of alignments receiving intermediate MAPQ scores (Fig. S3b). Thus, selecting at least a MAPQ > 10 1005 1006 threshold is necessary, but community standards may inform what intermediate threshold is chosen. In the replication timing and maize genomics communities, we have seen MAPQ thresholds ranging from MAPQ $>10$ to MAPQ $>30$. To plot the distribution of MAPQ scores present in alignment files, use the SAMtools view 1.9 app by selecting the Plot distribution of $M A P Q$ checkbox. Once a threshold is chosen, select the Use MAPQ 1010 filtering checkbox and enter the $M A P Q$ value to filter. These MAPQ filtering steps can be 
done simultaneously with the SAMtools view filtering described in section 3.10.3 or as a two-step process (Fig. S3).

1013

1014

1015

1016

1017

1018

1019

1020

1021

1022

1023

1024

1025

1026

1027

1028

1029

1030

1031

1032

1033

16. App Usage. Each app listed in this Repli-seq data pipeline (Table 1) follows the same general organization. Apps usually contain multiple dropdown sections, that include 1) Analysis name (Fig. S11), 2) Inputs, 3) Parameters/options 4) Output options. In the analysis name section, the specific name of the run and the location of the output folder can be modified. A general introduction to navigating and using apps in the DE can be found at https://cyverse.atlassian.net/wiki/spaces/DEmanual/overview.

17. Trim Galore! Parameters and Adapter Sequences. Trim Galore! is a wrapper tool that incorporates Cutadapt and FastQC to assess and remove low quality and adapter sequences from the sequencing read files (54). If inputting paired-end R1 and R2 fastq files, select the Paired checkbox on the Input dropdown. The Paired option removes entire read pairs together if one of the reads becomes too short after trimming. The default parameters are a good starting place. These defaults include trimming read ends with low quality base calls (parameter Quality - 20), the base pair overlap required for adapter trimming (parameter Stringency -1 ), the maximum error rate allowed (parameter Error rate - 0.1), and the length in base pairs below which to discard short reads after trimming (parameter Length - 20). Also select the fastqc checkbox to generate a FastQC quality control report. The Adapter sequence to be trimmed text box should include any known Illumina adapters and indexes included in the sequencing reads (84). If no adapter sequence is listed, Trim Galore! will auto-detect adapter sequences. The Trim Galore! (85) and FastQC (86) manuals provide additional parameter information and example reports for troubleshooting. 
18. Picard MarkDuplicates Parameters. The Picard-MarkDuplicates-2.7.1 app can locate and remove duplicate reads resulting from over amplification by PCR during library construction (PCR duplicates) or incorrect cluster identification on the sequencer (optical duplicates; (53)). The only parameters that need to be selected are Validation stringency lenient and the Remove duplicates checkbox to have the marked duplicates removed (Fig. S4).

19. Deeptools MultiBamSummary Parameters. The deeptools_3.2.0 app can accept up to ten .bam files as input. For our analysis, this has included filtered .bam files from three biological replicates each for early, middle and late S-phase slices. The bins parameter should be entered to correspond with the desired static bin size in base pairs. The Correlation type dropdown includes the Pearson's correlation coefficient and the nonparametric Spearman's rank correlation coefficient. We routinely use Correlation type - Pearson to compare biological replicates (Fig. S6).

20. Optional Random Downsampling. If desired, alignment files can be randomly downsampled to the desired coverage or percentage of reads retained. We recommend the usage of Picard DownsampleSam (53) and setting Probability $(-P)$ to the desired value. This tool is not available in the CyVerse DE and requires command line scripts.

21. Analysis Bin Size Selection. The Repliscan app aggregates read counts into static bins (windows) across the genome. The default Analysis bin size in base pairs setting is 1000 . However, this should be considered only as an effective minimum value. One should consider the EdU-labeling time (see Note 1), available sequencing depth, and the estimated size of a region of DNA that replicates from a single origin (replicon) in your system. Lower coverage data can be used at lower resolution by increasing the bin size. 
We recommend that the selected bin size should be at least an order of magnitude smaller than the estimated replicon size to detect transitions from one replication time to another. The average replicon size has been estimated as $47 \pm 13 \mathrm{~kb}$ for monocots and $66 \pm 11 \mathrm{~kb}$ for dicot plants (87).

22. Removing High and Low Coverage Bins. Genomic bins with extremely high coverage in the G1 control can be removed from the analysis in this step (see discussion below on the remove and percentile cutoff parameters). However, we find that doing so is not usually necessary because the subsequent step of dividing the scaled read numbers in each S1065 phase bin by the scaled read numbers in the corresponding G1 bin is sufficient to normalize high coverage "spikes" (Fig. 4). In contrast, statistical artifacts associated with division by small numbers can lead to 1068 spurious results for genomic bins that have extremely low read counts in the G1 control. To reduce the impact of such artifacts, low coverage bins can be removed from the analysis using the remove parameter. The default setting remove - norm represents a process in which a distribution of reads per bin in the G1 data are calculated, natural log transformed, and used to fit a normal distribution. This approach is suitable for distributions that are relatively symmetric and normal-like after the natural log transformation. From the fitted normal distribution, bins in the upper and lower tails can be removed using the dependent parameter percentile cutoff (default setting is 2.5-97.5). genome, these default settings may not be optimal. In our experience with maize data that has been filtered to remove alignments with low MAPQ (e.g. MAPQ < 10) and binned in 3-kb bins, the natural log transformed distribution of reads per bin has a highly negative 
skew (Fig. S10a). In this case, we recommend using the remove - percentile option where bins with a natural log transformed read count below a certain percentile threshold are removed, without fitting a probability distribution to the data. Selecting an appropriate percentile cutoff value is dependent on sequencing depth, bin size and the quality of the reference genome assembly. We recommend users try several cutoffs, but at a minimum, we recommend a percentile cutoff value that removes the lower $1 \%$ of bins in the reads per bin distribution (percentile cutoff - 1-100). It is important to note that this percentile cutoff parameter only applies to bins with some level of reads. G1 bins with zero reads will not be included in the plotted distribution (G1_coverage_cut.png; see Table 2 and Fig. S10a) because of the natural log transform and ratios with zero as the denominator are set to zero in the ratio calculation step (section 3.11.6).

23. Sequence Depth Scaling Based on Aggregate Method. If the default aggregate - sum parameter (section 3.11.3) is used, sequence depth scaling occurs after the biological replicates are summed and the G1 control bins with outlying read coverage are removed (section 3.11.4). However, if aggregate is set differently (e.g. mean or median), sequence depth scaling occurs twice, once before the biological replicates are aggregated and again after they are aggregated and the G1 reference bins with outlying read coverage are removed. This second scaling step accounts for the possibility of an unbalanced number of biological replicates.

24. Normalization to a Non-replicating Control. A properly sorted, truly non-replicating G1 control is important to avoid problems that may arise from copy number differences, mappability issues, and the presence of repeat sequences that are not fully represented in the reference genome. Pure G1 (definitively 2C) reference samples are easily obtained 
with the EdU procedure we describe, as nuclei at all stages of replication are well separated from the G1 population (Fig. 2). Similarly, clean separations of non-replicating reference populations cannot be obtained with techniques that do not physically separate labeled and unlabeled nuclei. When analyzing such data, including data obtained prior to the advent of EdU technology, it may be necessary to use a proxy for a truly nonreplicating control, such as a "total S pool" or DNA from an unsorted mixture of replicating and non-replicating nuclei. For examples and further discussion, see the analysis of pre-existing Drosophila and human Repli-seq data in (29). We do not recommend this procedure when proper G1 control data are available. When necessary,

Acknowledgements. This work was supported by grants from the NSF Plant Genome Research 1116 Program (NSF IOS-1025830 and IOS-2025811 to L.H.B.).

\section{Figure legends}

1119 Fig. 1 Repli-seq workflow. A simplified Repli-seq workflow, highlighting the major steps in the 1120 protocol. The dashed lines connect four of the text boxes to a picture or cartoon illustrating that 1121 step. The first picture is a 3-day old B73 maize seedling with a white box drawn around the 0-1 $1122 \mathrm{~mm}$ portion of the root tip, which is dissected and used in this protocol. The second picture is an 1123 example of a mini-food processor (not a full-sized blender) used for chopping fixed, frozen root 1124 tips for bulk nuclei isolation. The third picture shows an example of a bivariate plot from flow 1125 sorting EdU-labeled and DAPI stained nuclei, illustrating the S-phase "arc" (see Fig. 2). The last 
1126 picture is an example of the output of the Repli-seq data analysis pipeline using Repliscan for a

$1127 \sim 2 \mathrm{Mb}$ region on maize B73 chromosome 4. The blue, green, and red tracks represent normalized

1128 replication signal in early (E), middle (M) and late (L) portions of S-phase, respectively. The

1129 bottom track shows the final classification (segmentation) of replication time(s) at each locus 1130 (see Fig. 4).

1132 Fig. 2 Flow sorting gating strategy to remove debris and doublets, final sorting gates, and

1133 reanalysis of sorted, replicating nuclei. $(\mathbf{a}-\mathbf{c})$ The gating strategy used to separate debris and

1134 doublets from intact nuclei is illustrated in three bivariate pseudo-colored dot plots. Each dot is a

1135 single event and the color gradient (blue to red) represents increasing nuclear density. (a) Parent

1136 gate 1 (PG1) differentiates debris from intact nuclei based on light scattering properties (side

1137 scatter pulse height; SSC-H) and DNA content (DAPI fluorescence; DAPI-H). A gate (black

1138 polygon) is drawn around intact nuclei of all ploidy levels, excluding debris, and broken nuclei.

1139 (b) The nuclei in PG1 are further gated using Parent gate 2 (PG2) to remove doublets (aggregates 1140 of two or more nuclei). Doublets are differentiated from single nuclei using DAPI pulse width 1141 (DAPI-W) and DAPI pulse area (DAPI-A) to reflect particle geometry and size. (c) Nuclei in the 1142 singlet gate (PG2) are represented based on Alexa fluor 488 fluorescence (AF-488-H) and DNA 1143 content (DAPI-H). EdU/AF-488-labeled S-phase nuclei form an "arc" between the G1 and G2 1144 populations (2C and 4C DNA contents, respectively). Sorting gates (black rectangles) identify 1145 populations separated for replication timing analysis: G1 (non-replicating nuclei with 2C DNA 1146 content), early S phase (E; replicating nuclei with 2C DNA content), middle S phase (M;

1147 replicating nuclei with DNA content between $2 \mathrm{C}$ and $4 \mathrm{C}$ ) and late S phase (L; replicating nuclei 1148 with 4C DNA content). We do not normally sort G2 nuclei, as the G1 population is sufficient to 
1149 normalize for copy number and sequencability. Nuclei in the four gated fractions are sorted into

1150 individual tubes and their DNA is sequenced and analyzed as described in this protocol. (d)

1151 Overlaid univariate histograms of relative DNA content expressed as DAPI pulse height (DAPI-

$1152 \mathrm{H}$ ) showing a reanalysis of nuclei populations from the $\mathrm{E}$ (blue peak), $\mathrm{M}$ (green peak), and L (red

1153 peak) sorted populations in panel $\mathbf{c}$. The grey histogram shows all nuclei from PG2 for reference.

1154 The overlaid E, M, and L histograms demonstrate the relative purity of the sorted populations, as

1155 there is very little overlap between them. The overlap of the E and L peaks with the grey G1 and

1156 G2 peaks, respectively, emphasizes the benefit of using EdU labeling to differentiate replicating

1157 nuclei from non-replicating nuclei, achieving a sort purity nearly impossible if sorting on DNA

1158 content alone.

1159

1160 Fig. 3 Optimizing EdU pulse length. A flow cytometric analysis of an EdU-labeling time

1161 course is shown. Roots of 3-day old seedlings were pulse-labeled with $25 \mu \mathrm{M}$ EdU for 5, 10, and

$116220 \mathrm{~min}$. Nuclei were isolated from the terminal $0-1 \mathrm{~mm}$ of fixed root segments and analyzed by

1163 flow cytometry. The S-phase nuclei with incorporated EdU (above dashed line) can be clearly

1164 distinguished from the non-replicating, unlabeled G1 and G2 nuclei populations (below dashed

1165 line). At 5 min, a detectable "arc" of EdU labeled nuclei has already formed, but the arc is

1166 further differentiated from the G1 and G2 populations after at least $10 \mathrm{~min}$. We chose a $20 \mathrm{~min}$

1167 labeling time because of the clean separation of EdU-labeled nuclei from the unlabeled G1 and

1168 G2 populations for optimal sorting (see Note 1).

1169

1170 Fig. 4 Repliscan output files showing step by step analysis of Repli-seq data. (a-d) An

1171 example of Repliscan output for a 5-Mb region on chromosome 4 of the maize B73 genome, 
1172 presented in the Integrated Genomics Viewer (IGV) genome browser. The small red rectangle

1173 located above the genome coordinates represents the location on chromosome 4 of the

1174 highlighted 5-Mb region. The dark blue tick marks represent annotated genes in B73 RefGen_v4.

1175 (a) Scaled read densities in 3-kb static bins (windows) were calculated for the G1 non-replicating

1176 control (grey) and the early (E), middle (M), and late (L) S-phase samples. The G1 control data

1177 are used to normalize for sequencing biases, read mappability variation in the reference genome,

1178 and possible differences in copy number. Notice the corresponding "spikes" of high signal in the

1179 tracks for the G1 control (grey) and the late S-phase sample (red). (b) For each S-phase sample, a

1180 ratio to G1 was calculated by dividing the scaled read density in each S-phase bin by the

1181 corresponding scaled read density in the G1 control. Notice the absence of "spikes" in the late S-

1182 phase track after this G1 normalization (see Note 22). (c) Haar wavelet smoothing was

1183 performed to remove noise without altering peak boundaries (section 3.11.7). (d) Each locus was

1184 classified (segmentation) with a replication time(s). The segmented RT classes represented are as

1185 follows: early (blue), early-mid (teal), middle (green), mid-late (yellow), late (red) and not-

1186 segmented (white) (see section 3.11.8).

1188 Table 1. Repli-seq data analysis software workflow

\begin{tabular}{|c|c|c|}
\hline Analysis step & CyVerse application ${ }^{a}$ & $\begin{array}{l}\text { Software } \\
\text { reference(s) }\end{array}$ \\
\hline Read quality control and trimming & Trim-galore-0.4.1 & (54) \\
\hline Alignment & BWA_mem_0.7.15 & (51) \\
\hline Alignment filtering & samtools view 1.9 & $(51,55)$ \\
\hline Duplicate read removal & Picard-MarkDuplicates-2.7.1 & (53) \\
\hline Alignment statistics & Samtools 1.7 Flagstat ${ }^{\mathbf{b}}$ & (55) \\
\hline Correlation of biological replicates & deeptools_3.2.0 & (56) \\
\hline Replication timing and classification analysis & Repliscan 0.1.0 & (29) \\
\hline
\end{tabular}

${ }^{a}$ Available within the CyVerse Discovery Environment (https://de.cyverse.org/de/).

${ }^{b}$ The Samtools 1.7 Flagstat app can be run at multiple points in the analysis to assess the number of reads filtered out. 
Table 2. CyVerse applications input and output files

\begin{tabular}{|c|c|c|}
\hline $\begin{array}{l}\text { CyVerse } \\
\text { application }\end{array}$ & Input files & Output files $^{c}$ \\
\hline Trim-galore-0.4.1 & Sequencing fastq files & $\begin{array}{l}\text { Trimmed fastq files } \\
\text { FastQC reports and images }\end{array}$ \\
\hline BWA_mem_0.7.15 & $\begin{array}{l}\text { Reference genome } f a \text { file }^{\mathrm{a}} \\
\text { Trimmed fastq files }\end{array}$ & Alignment .sam file \\
\hline samtools view 1.9 & Alignment .sam or .bam file & $\begin{array}{l}\text { Filtered alignment .bam file } \\
\text { MAPQ score distribution plot.png }\end{array}$ \\
\hline $\begin{array}{l}\text { Picard- } \\
\text { MarkDuplicates- } \\
2.7 .1\end{array}$ & Filtered alignment . bam file & De-duplicated .bam file \\
\hline $\begin{array}{l}\text { Samtools } 1.7 \\
\text { Flagstat }\end{array}$ & $\begin{array}{l}\text { Any alignment . bam or .sam } \\
\text { file }\end{array}$ & alignment_stats.txt $\mathrm{f}^{\mathrm{f}}$ \\
\hline deeptools_3.2.0 & $\begin{array}{l}\text { Individual biological replicate } \\
\text { filtered, de-duplicated .bam } \\
\text { files }\end{array}$ & $\begin{array}{l}\text { Correlation heatmap multibam_heatmap.png } \\
\text { Correlation scatterplot multibam_scatter.png }\end{array}$ \\
\hline Repliscan 0.1.0 & $\begin{array}{l}\text { Reference genome } . f a \text { file }^{\mathrm{a}} \\
\text { Input configuration } \\
\text { input.txt file } \\
\text { CyVerse directory containing } \\
\text { filtered, de-duplicated } . \text { bam } \\
\text { files }\end{array}$ & $\begin{array}{l}\text { Merged, binned *.bedGraph files } \\
\text { Scaled, coverage cutoff removed *_norm.bedGraph files } \\
\text { Ratio to G1*_ratio.bedGraph files } \\
\text { Smoothed *_ratio_3.smooth.bedGraph files } \\
\text { RT class segmentation ratio_segmentation.gff } 3^{\mathrm{h}} \\
\text { Optional 'plot'files: } \\
\text { G1 reads per bin distribution G1_coverage_cut.png }{ }^{\mathrm{i}} \\
\text { Repliscan parameters and thresholds .out file }{ }^{\mathrm{i}} \\
\text { Segmentation threshold * fig.png files } \\
\text { Error log .err file }\end{array}$ \\
\hline
\end{tabular}

${ }^{\mathrm{a}}$ For example, the Zea mays B73 RefGen_v4 genome is hosted in the CyVerse Data Commons (/iplant/home/shared/iplantcollaborative/genomeservices/builds/1.0.0/24_77/Zea.AGPv4/de_support) as well as many other reference genomes.

${ }^{\mathrm{b}}$ See Fig. S9a.

${ }^{\mathrm{c}}$ Each application also produces various kinds of error and/or log files to help troubleshoot possible problems.

${ }^{\mathrm{d}}$ See FastQC manual ((86).

e See Fig. S3b.

${ }^{\mathrm{f}}$ See Table 3 for example.

g See Fig. S6b.

${ }^{\mathrm{h}}$ See Fig. 4.

${ }^{\mathrm{i}}$ See Fig. S10a.

\section{Table 3. An example of Repli-seq sequence read processing statistics}

\begin{tabular}{|c|c|c|c|c|c|}
\hline \multirow[b]{2}{*}{$\begin{array}{l}\text { Repli-seq } \\
\text { sample }^{\text {a }}\end{array}$} & \multicolumn{4}{|c|}{ Number of reads } & \multirow[b]{2}{*}{$\begin{array}{l}\% \text { Uniquely } \\
\text { aligned of total }\end{array}$} \\
\hline & $\begin{array}{l}\text { Total } \\
\text { sequenced }\end{array}$ & $\begin{array}{l}\text { Properly paired, } \\
\text { primary alignment }^{b}\end{array}$ & $\begin{array}{l}\text { Duplicates } \\
\text { removed }^{c}\end{array}$ & $\begin{array}{l}\text { Unique } \\
(\text { MAPQ }>10)^{d}\end{array}$ & \\
\hline G1 control & $189,439,280$ & $178,771,352$ & $167,040,024$ & $119,162,753$ & 62.9 \\
\hline Early S & $396,856,512$ & $372,022,064$ & $362,369,380$ & $261,289,352$ & 65.8 \\
\hline Middle S & $384,595,762$ & $348,490,682$ & $340,764,126$ & $241,379,234$ & 62.8 \\
\hline Late $\mathrm{S}$ & $220,400,974$ & $201,215,586$ & $193,557,372$ & $136,945,173$ & 62.1 \\
\hline
\end{tabular}

${ }^{a}$ Sequencing data represented in this table can be accessed at the NCBI SRA accession PRJNA327875.

${ }^{b}$ Aligned read counts after filtering out reads not in proper pairs and reads associated with secondary alignment locations.

${ }^{\mathrm{c}}$ Aligned read counts after removing duplicate reads. 
${ }^{\mathrm{d}}$ Aligned read counts for reads that are confidently mapped to a single location (unique), as assessed by BWA-MEM mapping quality (MAPQ) score. The maize genome has a large proportion of repetitive DNA, making unique alignments imperative. The percentage of unique reads in a Repli-seq dataset may vary widely depending on the repeat content and organization of the genome that is being analyzed.

\section{Supplemental Figure Legends}

1194 Fig. S1 Trim Galore! parameter window. The default parameters are entered, as well the 1195 fastqc checkbox selected to generate the FASTQC report. Any known Illumina adapters and 1196 index sequences should be entered in the Adapter sequence to be trimmed textbox. Trim Galore! 1197 will autodetect adapters if this parameter is left blank.

Fig. S2 BWA-MEM parameters and output options windows. The BWA-MEM default parameters (a) and default output options (b) are auto-populated.

Fig. S3 SAMtools view parameters window. (a) The SAMtools view app recommended quality (MAPQ) distribution found in the alignment file before filtering.

1207 marking and removing duplicate read alignments.

Fig. S5 SAMtools Flagstat output window. SAMtools Flagstat generates mapping statistics

1210 outputted as a nameable .txt file.

1212 Fig. S6 DeepTools multiBamSummary parameters window. (a) The recommended

1213 Correlation type is selected. A desired static bin size (bins in base pairs) should also be selected. 
1214 (b) The Pearson correlation coefficients between individual biological replicates for early, 1215 middle and late $\mathrm{S}$ samples represented as a heatmap with a hierarchical clustering dendrogram.

1217 Fig. S7 Repliscan default parameters. The Repliscan app default parameters are auto1218 populated. Select the plot checkbox to generate additional output files (see Table 2 and section 1219 3.11.9).

1221 Fig. S8 Repliscan recommended parameters for maize B73 test dataset. For the maize test 1222 dataset, which has been filtered to remove alignments with MAPQ $<10$ and randomly

1223 downsampled, we recommend adjusting the Analysis bin size in base pairs as well as the remove 1224 parameter with the dependent parameter percentile cutoff. These setting adjustments are shown 1225 in the parameter window. See Note 21 and 22 for a detailed description.

1227 Fig. S9 Repliscan input configuration example. (a) An input.txt configuration file is needed to 1228 assign individual files to analysis name labels. Individual files should be delimited by a tab. The 1229 name labels are used in output file naming and RT class segmentation naming. (b) Repliscan 1230 Inputs window requiring reference genome in Fasta format, a configuration file with a list of 1231 .bam files (input.txt), and a directory containing the .bam files.

1233 Fig. S10 Repliscan optional output example. Selecting the plot checkbox generates additional 1234 output files. (a) Included in these output files is a plot of the distribution of natural log 1235 transformed reads per bin in the G1 data and the selected cutoff (grey shaded area) from the 1236 remove and percentile cutoff parameters. For maize data that has been filtered to remove 
alignments with MAPQ $<10$, the distribution is negatively skewed (see Note 22). (b) An

example of the .out file, which includes the parameter settings used and the auto-tuned RT class segmentation thresholds for individual chromosomes (see section 3.11.8). The plots shown represent the files from the maize B73 test dataset with recommended parameters (Fig. S8).

1242 Fig. S11 Analysis Name dropdown window example. Each app listed in the Repli-seq data analysis pipeline (Table 1) follows the same general organization. In the Analysis Name section,

1244 the specific name of the run and the location of the output folder can be modified.

\section{References}

1. Klein KN, Gilbert DM (2016) Epigenetic vs. sequence-dependent control of eukaryotic replication timing. In: D Kaplan (ed) The initiation of DNA replication in eukaryotes. Springer International Publishing, Switzerland, pp 39-63

2. Marchal C, Sima J, Gilbert DM (2019) Control of DNA replication timing in the 3D genome. Nat Rev Mol Cell Biol 20(12):721-737

3. Gilbert DM, Takebayashi S-I, Ryba T, Lu J, Pope BD, Wilson KA, Hiratani I (2010) Space and time in the nucleus: developmental control of replication timing and chromosome architecture. Cold Spring Harb Symp Quant Biol 75:143-153

4. Schubeler D, Scalzo D, Kooperberg C, van Steensel B, Delrow J, Groudine M (2002) Genome-wide DNA replication profile for Drosophila melanogaster: a link between transcription and replication timing. Nat Genet 32(3):438-442

5. Woodfine K, Fiegler H, Beare DM, Collins JE, McCann OT, Young BD, Debernardi S, Mott R, Dunham I, Carter NP (2004) Replication timing of the human genome. Hum Mol Genet 13(2):191-202

6. Hiratani I, Gilbert DM (2009) Replication timing as an epigenetic mark. Epigenetics 4(2):93-97

7. Schwaiger M, Stadler MB, Bell O, Kohler H, Oakeley EJ, Schubeler D (2009) Chromatin state marks cell-type- and gender-specific replication of the Drosophila genome. Genes Dev 23(5):589-601

8. Hansen RS, Thomas S, Sandstrom R, Canfield TK, Thurman RE, Weaver M, Dorschner MO, Gartler SM, Stamatoyannopoulos JA (2010) Sequencing newly replicated DNA reveals widespread plasticity in human replication timing. Proc Natl Acad Sci U S A 107(1):139-144

9. Eaton ML, Prinz JA, MacAlpine HK, Tretyakov G, Kharchenko PV, MacAlpine DM (2011) Chromatin signatures of the Drosophila replication program. Genome Res 21(2):164-174 
10. Lubelsky Y, Prinz JA, DeNapoli L, Li Y, Belsky JA, MacAlpine DM (2014) DNA replication and transcription programs respond to the same chromatin cues. Genome Res 24(7):1102-1114

11. Gilbert DM (2002) Replication timing and transcriptional control: beyond cause and effect. Curr Opin in Cell Biol 14:377-383

12. Ryba T, Hiratani I, Lu J, Itoh M, Kulik M, Zhang J, Schulz TC, Robins AJ, Dalton S, Gilbert DM (2010) Evolutionarily conserved replication timing profiles predict longrange chromatin interactions and distinguish closely related cell types. Genome Res 20(6):761-770

13. Yaffe E, Farkash-Amar S, Polten A, Yakhini Z, Tanay A, Simon I (2010) Comparative analysis of DNA replication timing reveals conserved large-scale chromosomal architecture. PLoS Genet 6(7):e1001011

14. Pope BD, Ryba T, Dileep V, Yue F, Wu W, Denas O, Vera DL, Wang Y, Hansen RS, Canfield TK, Thurman RE, Cheng Y, Gulsoy G, Dennis JH, Snyder MP, Stamatoyannopoulos JA, Taylor J, Hardison RC, Kahveci T, Ren B, Gilbert DM (2014) Topologically associating domains are stable units of replication-timing regulation. Nature 515(7527):402-405

15. Rivera-Mulia JC, Buckley Q, Sasaki T, Zimmerman J, Didier RA, Nazor K, Loring JF, Lian Z, Weissman S, Robins AJ, Schulz TC, Menendez L, Kulik MJ, Dalton S, Gabr H, Kahveci T, Gilbert DM (2015) Dynamic changes in replication timing and gene expression during lineage specification of human pluripotent stem cells. Genome Res 25(8):1091-1103

16. Agier N, Delmas S, Zhang Q, Fleiss A, Jaszczyszyn Y, van Dijk E, Thermes C, Weigt M, Cosentino-Lagomarsino M, Fischer G (2018) The evolution of the temporal program of genome replication. Nat Commun 9(1):2199

17. Thorpe SD, Charpentier M (2017) Highlight on the dynamic organization of the nucleus. Nucleus 8(1):2-10

18. Shultz RW, Tatineni VM, Hanley-Bowdoin L, Thompson WF (2007) Genome-wide analysis of the core DNA replication machinery in the higher plants Arabidopsis and rice. Plant Physiol 144(4):1697-1714

19. Gnan S, Flyamer IM, Klein KN, Castelli E, Rapp A, Maiser A, Chen N, Weber P, Enervald E, Cardoso MC, Bickmore WA, Gilbert DM, Buonomo SCB (2020) Nuclear organisation and replication timing are coupled through RIF1-PP1 interaction. bioRxiv: https://doi.org/10.1101/812156

20. Sreesankar E, Senthilkumar R, Barathi V, Mishra R, Mishra K (2012) Functional diversification of yeast telomere associated protein, Rif1, in higher eukaryotes. BMC Genom 13:255

21. Wheeler E, Brooks AM, Concia L, Vera DL, Wear EE, LeBlanc C, Ramu U, Vaughn MW, Bass HW, Martienssen RA, Thompson WF, Hanley-Bowdoin L (2020) Arabidopsis DNA replication initiates in intergenic, AT-rich open chromatin. Plant Physiol 183(1):206-220

22. Bass HW, Hoffman GG, Lee TJ, Wear EE, Joseph SR, Allen GC, Hanley-Bowdoin L, Thompson WF (2015) Defining multiple, distinct, and shared spatiotemporal patterns of DNA replication and endoreduplication from 3D image analysis of developing maize (Zea mays L.) root tip nuclei. Plant Mol Biol 89(4-5):339-351 
1319

1320

1321

1322

1323

1324

1325

1326

1327

1328

1329

1330

1331

1332

1333

1334

1335

1336

1337

1338

1339

1340

1341

1342

1343

1344

1345

1346

1347

1348

1349

1350

1351

1352

1353

1354

1355

1356

1357

1358

1359

1360

1361

1362

1363

23. Savadel SD, Bass HW (2017) Take a look at plant DNA replication: recent insights and new questions. Plant Signal Behav 12(4):e1311437

24. Mickelson-Young L, Wear E, Mulvaney P, Lee TJ, Szymanski ES, Allen G, HanleyBowdoin L, Thompson W (2016) A flow cytometric method for estimating S-phase duration in plants. J Exp Bot 67(21):6077-6087

25. Lee TJ, Pascuzzi PE, Settlage SB, Shultz RW, Tanurdzic M, Rabinowicz PD, Menges M, Zheng P, Main D, Murray JA, Sosinski B, Allen GC, Martienssen RA, Hanley-Bowdoin L, Vaughn MW, Thompson WF (2010) Arabidopsis thaliana chromosome 4 replicates in two phases that correlate with chromatin state. PLoS Genet 6(6):e1000982

26. Wear EE, Song J, Zynda GJ, LeBlanc C, Lee TJ, Mickelson-Young L, Concia L, Mulvaney P, Szymanski ES, Allen GC, Martienssen RA, Vaughn MW, Hanley-Bowdoin L, Thompson WF (2017) Genomic analysis of the DNA replication timing program during mitotic S phase in maize (Zea mays) root tips. Plant Cell 29(9):2126-2149

27. Concia L, Brooks AM, Wheeler E, Zynda GJ, Wear EE, LeBlanc C, Song J, Lee TJ, Pascuzzi PE, Martienssen RA, Vaughn MW, Thompson WF, Hanley-Bowdoin L (2018) Genome-wide analysis of the Arabidopsis replication timing program. Plant Physiol 176(3):2166-2185

28. Wear EE, Song J, Zynda GJ, Mickelson-Young L, LeBlanc C, Lee TJ, Deppong DO, Allen GC, Martienssen RA, Vaughn MW, Hanley-Bowdoin L, Thompson WF (2020) Comparing DNA replication programs reveals large timing shifts at centromeres of endocycling cells in maize roots. PLoS Genet 16(10):e1008623

29. Zynda GJ, Song JW, Concia L, Wear EE, Hanley-Bowdoin L, Thompson WF, Vaughn MW (2017) Repliscan: a tool for classifying replication timing regions. BMC Bioinform 18:1-14

30. Bass HW, Wear EE, Lee TJ, Hoffman GG, Gumber HK, Allen GC, Thompson WF, Hanley-Bowdoin L (2014) A maize root tip system to study DNA replication programmes in somatic and endocycling nuclei during plant development. J Exp Bot 65(10):2747-2756

31. Galbraith DW, Harkins KR, Maddox JM, Ayres NM, Sharma DP, Firoozabady E (1983) Rapid flow cytometric analysis of the cell cycle in intact plant tissues. Science 220(4601):1049-1051

32. Wear EE, Concia L, Brooks AM, Markham EA, Lee TJ, Allen GC, Thompson WF, Hanley-Bowdoin L (2016) Isolation of plant nuclei at defined cell cycle stages using EdU labeling and flow cytometry. Methods Mol Biol 1370:69-86

33. Salic A, Mitchison TJ (2008) A chemical method for fast and sensitive detection of DNA synthesis in vivo. Proc Natl Acad Sci USA 105(7):2415-2420

34. Kotogany E, Dudits D, Horvath GV, Ayaydin F (2010) A rapid and robust assay for detection of S-phase cell cycle progression in plant cells and tissues by using ethynyl deoxyuridine. Plant Methods 6(5)

35. Dolezel J, Cihalikova J, Weiserova J, Lucretti S (1999) Cell cycle synchronization in plant root meristems. Methods Cell Sci 21:95-107

36. Planchais S, Glab N, Inze D, Bergounioux C (2000) Chemical inhibitors: a tool for plant cell cycle studies. FEBS Lett 476:78-83

37. Menges M, Murray JA (2002) Synchronous Arabidopsis suspension cultures for analysis of cell-cycle gene activity. Plant J 30(2):203-212 
1364

1365

1366

1367

1368

1369

1370

1371

1372

1373

1374

1375

1376

1377

1378

1379

1380

1381

1382

1383

1384

1385

1386

1387

1388

1389

1390

1391

1392

1393

1394

1395

1396

1397

1398

1399

1400

1401

1402

1403

1404

1405

1406

1407

38. Lee M, Phillips RL (1988) The chromosomal basis of somaclonal variation. Annu Rev of Plant Physiol and Plant Mol Biol 39:413-437

39. Phillips RL, Kaeppler SM, Olhoft P (1994) Genetic instability of plant tissue cultures: breakdown of normal controls. Proc Natl Acad Sci USA 91(12):5222-5226

40. Tanurdzic M, Vaughn MW, Jiang H, Lee TJ, Slotkin RK, Sosinski B, Thompson WF, Doerge RW, Martienssen RA (2008) Epigenomic consequences of immortalized plant cell suspension culture. PLoS Biol 6(12):2880-2895

41. Dolezel J, Greilhuber J, Suda J (2007) Estimation of nuclear DNA content in plants using flow cytometry. Nat Protoc 2(9):2233-2244

42. Gendrel AV, Lippman Z, Martienssen R, Colot V (2005) Profiling histone modification patterns in plants using genomic tiling microarrays. Nat Methods 2(3):213-218

43. Galbraith DW, Anderson MT, Herzenberg LA (1999) Flow cytometric analysis and FACS sorting of cells based on GFP accumulation. Methods Cell Biol 58:315-341

44. Galbraith DW, Bartos J, Dolezel J (2005) Flow cytometry and cell sorting in plant biotechnology. In: LA Skylar (ed) Flow Cytometry for Biotechnology. Oxford University Press, New York, pp 291-322

45. McCoy J (2002) Basic principles of flow cytometry. Hematology/oncology clinics of North America 16(2):229-243

46. Shapiro H (2003) Practical Flow Cytometry. Fourth ed. Wiley-Liss, Hoboken, NJ

47. Picot J, Guerin CL, Le Van Kim C, Boulanger CM (2012) Flow cytometry: retrospective, fundamentals and recent instrumentation. Cytotechnology 64(2):109-130

48. Darzynkiewicz Z, Zhao H (2014) Cell cycle analysis by flow cytometry. In: Encyclopedia of Life Sciences. John Wiley \& Sons, Ltd, Chichester

49. Wersto RP, Chrest FJ, Leary JF, Morris C, Stetler-Stevenson M, Gabrielson E (2001) Doublet discrimination in DNA cell-cycle analysis. Cytometry 46(5):296-306

50. Illumina (2017) Best practices for manually normalizing library concentrations. Available via https://support.illumina.com/bulletins/2017/03/best-practices-for-manuallynormalizing-library-concentrations.html. Accessed 21 Oct 2020

51. Li H (2013) Aligning sequence reads, clone sequences and assembly contigs with BWAMEM. ArXiv 1303

52. Li H, Ruan J, Durbin R (2008) Mapping short DNA sequencing reads and calling variants using mapping quality scores. Genome Res 18(11):1851-1858

53. Broad-Institute (2016) Picard: a set of command line tools (in Java) for manipulating high-throughput sequencing (HTS) data and formats such as SAM/BAM/CRAM and VCF. Available via https://broadinstitute.github.io/picard/. Accessed 20 Oct 2020

54. Krueger F (2012) Trim Galore!: a wrapper tool around Cutadapt and FastQC to consistently apply quality and adapter trimming to FastQ files. Available via https://www.bioinformatics.babraham.ac.uk/projects/trim galore/. Accessed 20 Oct 2020

55. Li H, Handsaker B, Wysoker A, Fennell T, Ruan J, Homer N, Marth G, Abecasis G, Durbin R, Genome Project Data Processing S (2009) The sequence alignment/map format and SAMtools. Bioinformatics 25(16):2078-9

56. Ramirez F, Dundar F, Diehl S, Gruning BA, Manke T (2014) deepTools: a flexible platform for exploring deep-sequencing data. Nucleic Acids Res 42(Web Server issue):W187-W191 
57. Merchant N, Lyons E, Goff S, Vaughn M, Ware D, Micklos D, Antin P (2016) The iPlant collaborative: cyberinfrastructure for enabling data to discovery for the life sciences. PLoS Biol 14(1):e1002342

58. Diaz A, Park K, Lim DA, Song JS (2012) Normalization, bias correction, and peak calling for ChIP-seq. Stat Appl Genet Mol Biol 11(3):Article 9

59. Percival DB, Walden AT (2000) Wavelet methods for time series analysis. Cambridge University Press, Cambridge, UK

60. Diermeier-Daucher S, Clarke ST, Hill D, Vollmann-Zwerenz A, Bradford JA, Brockhoff G (2009) Cell type specific applicability of 5-ethynyl-2'-deoxyuridine (EdU) for dynamic proliferation assessment in flow cytometry. Cytometry A 75(6):535-546

61. Kohlmeier F, Maya-Mendoza A, Jackson DA (2013) EdU induces DNA damage response and cell death in mESC in culture. Chromosome Res 21(1):87-100

62. Ross HH, Rahman M, Levkoff LH, Millette S, Martin-Carreras T, Dunbar EM, Reynolds BA, Laywell ED (2011) Ethynyldeoxyuridine (EdU) suppresses in vitro population expansion and in vivo tumor progression of human glioblastoma cells. J Neurooncol 105(3):485-498

63. Zhao H, Halicka HD, Li JW, Biela E, Berniak K, Dobrucki J, Darzynkiewicz Z (2013) DNA damage signaling, impairment of cell cycle progression, and apoptosis triggered by 5-Ethynyl-2 '-deoxyuridine incorporated into DNA. Cytometry A 83(11):979-988

64. Baluska F (1990) Nuclear size, DNA content, and chromatin condensation are different in individual tissues of the maize root apex. Protoplasma 158(1-2):45-52

65. Baluska F, Mancuso S (2013) Root apex transition zone as oscillatory zone. Front Plant Sci 4:Article 354

66. Rostovtsev VV, Green LG, Fokin VV, Sharpless KB (2002) A stepwise huisgen cycloaddition process: copper(I)-catalyzed regioselective "ligation" of azides and terminal alkynes. Angewandte Chemie International Edition 41(14):2596-2599

67. Endaya B, Cavanagh B, Alowaidi F, Walker T, de Pennington N, Ng JM, Lam PY, Mackay-Sim A, Neuzil J, Meedeniya AC (2016) Isolating dividing neural and brain tumour cells for gene expression profiling. J Neurosci Methods 257:121-133

68. Prutz W, Butler J, Land E (1990) Interaction of copper(I) with nucleic acids. International J Radiat Biol 58(2)

69. Soares E, Hebbelinck K, Soares H (2003) Toxic effects caused by heavy metals in the yeast Saccharomyces cerevisiae: a comparative study. Can J Microbiol 49:336-343

70. Galbraith DW (1989) Analysis of higher plants by flow cytometry and cell sorting. Int Rev Cytol 116:165-228

71. Bauer K (1993) Quality control issues in DNA content flow cytometry. Ann NY Acad Sci 677:59-77

72. Houtz B, Trotter J, Sasaki D (2004) BD FACService technotes: customer focused solutions. Customer Focused Solutions. Available via https://static.bdbiosciences.com/documents/BD Research Sorting TechBulletin.pdf. Accessed 22 Oct 2020

73. Schnable PS, Ware D, Fulton RS, Stein JC, Wei F, Pasternak S, Liang C, Zhang J, Fulton L, Graves TA, Minx P, Reily AD, Courtney L, Kruchowski SS, Tomlinson C, Strong C, Delehaunty K, Fronick C, Courtney B, Rock SM, Belter E, Du F, Kim K, Abbott RM, Cotton M, Levy A, Marchetto P, Ochoa K, Jackson SM, Gillam B, Chen W, Yan L, Higginbotham J, Cardenas M, Waligorski J, Applebaum E, Phelps L, Falcone J, Kanchi 
K, Thane T, Scimone A, Thane N, Henke J, Wang T, Ruppert J, Shah N, Rotter K, Hodges J, Ingenthron E, Cordes M, Kohlberg S, Sgro J, Delgado B, Mead K, Chinwalla A, Leonard S, Crouse K, Collura K, Kudrna D, Currie J, He R, Angelova A, Rajasekar S, Mueller T, Lomeli R, Scara G, Ko A, Delaney K, Wissotski M, Lopez G, Campos D, Braidotti M, Ashley E, Golser W, Kim H, Lee S, Lin J, Dujmic Z, Kim W, Talag J, Zuccolo A, Fan C, Sebastian A, Kramer M, Spiegel L, Nascimento L, Zutavern T, Miller B, Ambroise C, Muller S, Spooner W, Narechania A, Ren L, Wei S, Kumari S, Faga B, Levy MJ, McMahan L, Van Buren P, Vaughn MW, Ying K, Yeh CT, Emrich SJ, Jia Y, Kalyanaraman A, Hsia AP, Barbazuk WB, Baucom RS, Brutnell TP, Carpita NC, Chaparro C, Chia JM, Deragon JM, Estill JC, Fu Y, Jeddeloh JA, Han Y, Lee H, Li P, Lisch DR, Liu S, Liu Z, Nagel DH, McCann MC, SanMiguel P, Myers AM, Nettleton D, Nguyen J, Penning BW, Ponnala L, Schneider KL, Schwartz DC, Sharma A, Soderlund C, Springer NM, Sun Q, Wang H, Waterman M, Westerman R, Wolfgruber TK, Yang L, Yu Y, Zhang L, Zhou S, Zhu Q, Bennetzen JL, Dawe RK, Jiang J, Jiang N, Presting GG, Wessler SR, Aluru S, Martienssen RA, Clifton SW, McCombie WR, Wing RA, Wilson RK (2009) The B73 maize genome: complexity, diversity, and dynamics. Science 326(5956):1112-1115

74. Head SR, Komori HK, LaMere SA, Whisenant T, Van Nieuwerburgh F, Salomon DR, Ordoukhanian P (2014) Library construction for next-generation sequencing: overviews and challenges. Biotechniques 56(2):61—passim

75. 10x Genomics (2016) SPRIselect: DNA ratios affect the size range of library fragments. Available via https://support.10xgenomics.com/genome-exome/index/doc/technical-notespriselectdna-ratios-affect-the-size-range-of-library-fragments. Accessed 21 Oct 2020

76. Farouni R, Djambazian H, Ferri LE, Ragoussis J, Najafabadi HS (2020) Model-based analysis of sample index hopping reveals its widespread artifacts in multiplexed singlecell RNA-sequencing. Nat Commun 11(1):2704

77. Illumina (2017) Effects of index misassignment on multiplexing and downstream analysis. Understanding unique dual indexes (UDI) and associated library prep kits Available via https://support.illumina.com/bulletins/2018/08/understanding-unique-dualindexes--udi--and-associated-library-p.html. Accessed 21 Oct 2020

78. MacConaill LE, Burns RT, Nag A, Coleman HA, Slevin MK, Giorda K, Light M, Lai K, Jarosz M, McNeill MS, Ducar MD, Meyerson M, Thorner AR (2018) Unique, dualindexed sequencing adapters with UMIs effectively eliminate index cross-talk and significantly improve sensitivity of massively parallel sequencing. BMC Genom 19(1):30

79. Illumina (2018) Understanding unique dual indexes (UDI) and associated library prep kits. Available via https://support.illumina.com/bulletins/2018/08/understanding-uniquedual-indexes--udi--and-associated-library-p.html. Accessed 21 Oct 2020

80. Illumina (2016) Converting $\mathrm{ng} / \mathrm{ul}$ to $\mathrm{nM}$ when calculating dsDNA library concentration. Available via https://support.illumina.com/bulletins/2016/11/converting-ngl-to-nm-whencalculating-dsdna-library-concentration-.html. Accessed 21 Oct 2020

81. Bronner IF, Quail MA, Swerdlow H, Turner DJ (2009) Improved protocols for the Illumina genome analyzer sequencing system. Curr Protoc Hum Genet 18:10.1002/0471142905.hg1802s62

82. Arabidopsis Genome Initiative (2000) Analysis of the genome sequence of the flowering plant Arabidopsis thaliana. Nature 408(6814):796-815 
1500

1501

1502

1503

1504

1505

1506

1507

1508

1509

1510

1511

1512

1513

1514

1515

83. Lee H, Lee KW, Lee T, Park D, Chung J, Lee C, Park WY, Son DS (2018) Performance evaluation method for read mapping tool in clinical panel sequencing. Genes Genomics 40(2):189-197

84. Illumina (2020) Illumina adapter sequences. Available via https://support.illumina.com/downloads/illumina-adapter-sequences-document1000000002694.html. Accessed 21 Oct 2020

85. Krueger F (2019) Taking appropriate QC measure for RRBS-type or other -Seq application with Trim Galore! Available via:

https://github.com/FelixKrueger/TrimGalore/blob/master/Docs/Trim Galore User Guid e.md. Accessed 21 Oct 2020

86. Andrews S (2017) FastQC a quality control application for high throughput sequence data. Available via: https://www.bioinformatics.babraham.ac.uk/projects/fastqc/). Accessed 20 Oct 2020

87. Van't Hof J (1996) DNA replication in plants. In: DNA replication in eukaryotic cells. Cold Spring Harbor Press, Cold Spring Harbor, NY 
Figure 1

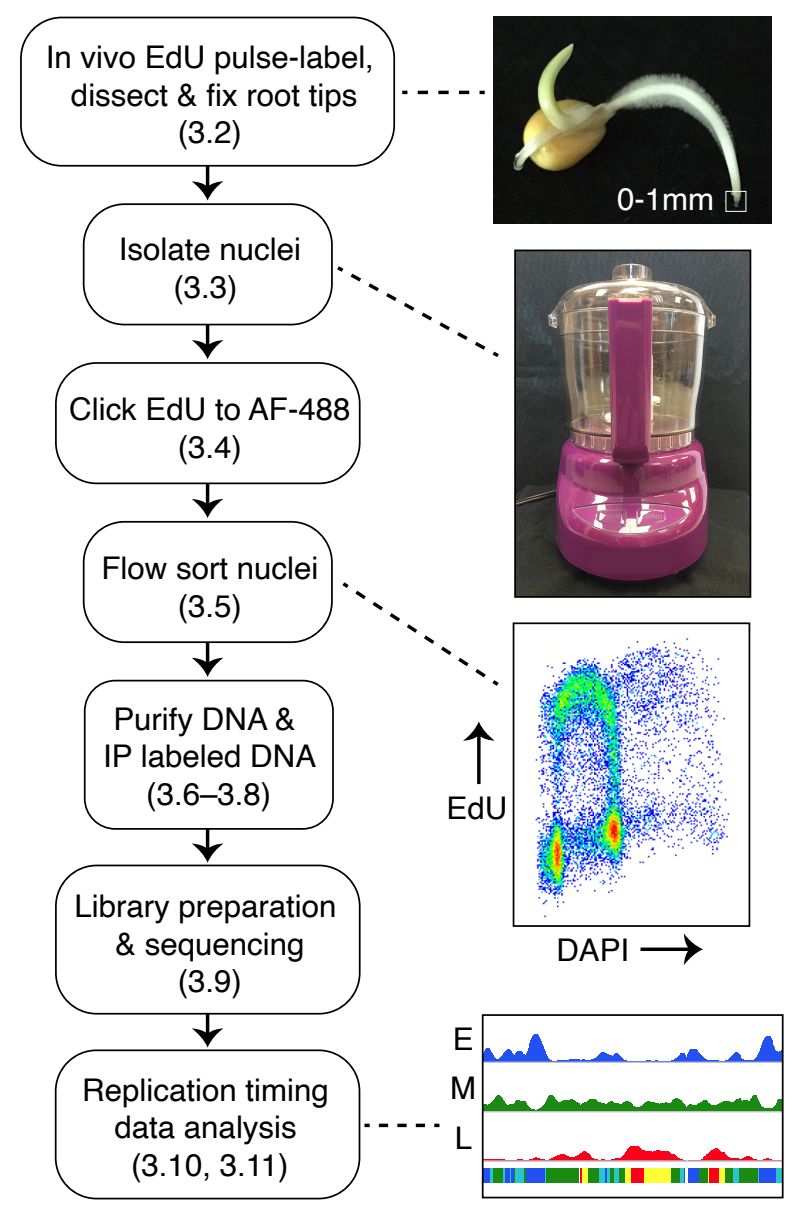

Fig. 1 Repli-seq workflow. A simplified Repli-seq workflow, highlighting the major steps in the protocol. The dashed lines connect four of the text boxes to a picture or cartoon illustrating that step. The first picture is a 3-day old B73 maize seedling with a white box drawn around the 0-1 mm portion of the root tip, which is dissected and used in this protocol. The second picture is an example of a mini-food processor (not a full-sized blender) used for chopping fixed, frozen root tips for bulk nuclei isolation. The third picture shows an example of a bivariate plot from flow sorting EdU-labeled and DAPI stained nuclei, illustrating the S-phase "arc" (see Fig. 2). The last picture is an example of the output of the Repli-seq data analysis pipeline using Repliscan for a $\sim 2 \mathrm{Mb}$ region on maize B73 chromosome 4 . The blue, green, and red tracks represent normalized replication signal in early $(E)$, middle $(M)$ and late $(L)$ portions of S-phase, respectively. The bottom track shows the final classification (segmentation) of replication time(s) at each locus (see Fig. 4). 
Figure 2

a
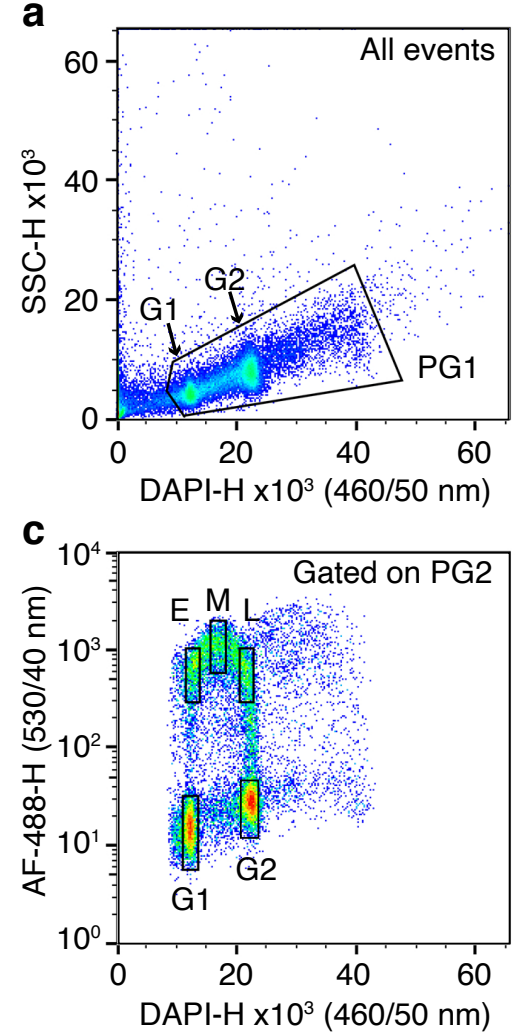

b

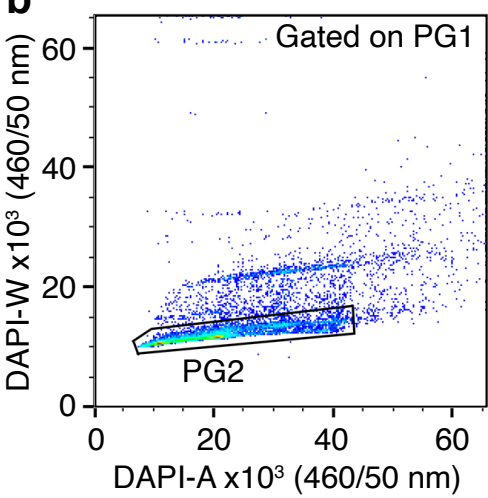

d

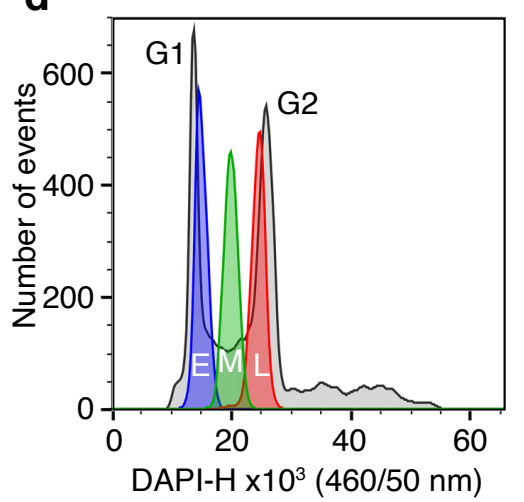

Fig. 2 Flow sorting gating strategy to remove debris and doublets, final sorting gates, and reanalysis of sorted, replicating nuclei. (a-c) The gating strategy used to separate debris and doublets from intact nuclei is illustrated in three bivariate pseudo-colored dot plots. Each dot is a single event and the color gradient (blue to red) represents increasing nuclear density. (a) Parent gate 1 (PG1) differentiates debris from intact nuclei based on light scattering properties (side scatter pulse height; SSC-H) and DNA content (DAPI fluorescence; DAPI-H). A gate (black polygon) is drawn around intact nuclei of all ploidy levels, excluding debris, and broken nuclei. (b) The nuclei in PG1 are further gated using Parent gate 2 (PG2) to remove doublets (aggregates of two or more nuclei). Doublets are differentiated from single nuclei using DAPI pulse width (DAPI-W) and DAPI pulse area (DAPI-A) to reflect particle geometry and size. (c) Nuclei in the singlet gate (PG2) are represented based on Alexa fluor 488 fluorescence (AF-488-H) and DNA content (DAPI-H). EdU/AF-488-labeled S-phase nuclei form an "arc" between the G1 and G2 populations (2C and 4C DNA contents, respectively). Sorting gates (black rectangles) identify populations separated for replication timing analysis: G1 (non-replicating nuclei with 2C DNA content), early S phase (E; replicating nuclei with $2 \mathrm{C}$ DNA content), middle S phase (M; replicating nuclei with DNA content between $2 \mathrm{C}$ and $4 \mathrm{C}$ ) and late $S$ phase (L; replicating nuclei with 4C DNA content). We do not normally sort G2 nuclei, as the G1 population is sufficient to normalize for copy number and sequencability. Nuclei in the four gated fractions are sorted into individual tubes and their DNA is sequenced and analyzed as described in this protocol. (d) Overlaid univariate histograms of relative DNA content expressed as DAPI pulse height (DAPI-H) showing a reanalysis of nuclei populations from the $E$ (blue peak), $\mathrm{M}$ (green peak), and L (red peak) sorted populations in panel c. The grey histogram shows all nuclei from PG2 for reference. The overlaid $E, M$, and $L$ histograms demonstrate the relative purity of the sorted populations, as there is very little overlap between them. The overlap of the $E$ and $L$ peaks with the grey G1 and G2 peaks, respectively, emphasizes the benefit of using EdU labeling to differentiate replicating nuclei from non-replicating nuclei, achieving a sort purity nearly impossible if sorting on DNA content alone. 
Figure 3

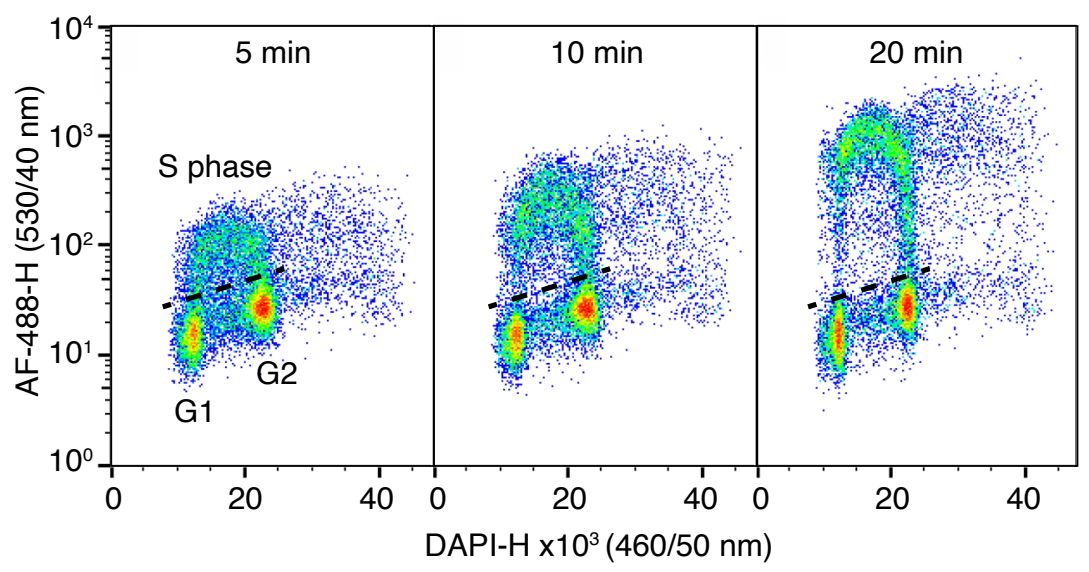

Fig. 3 Optimizing EdU pulse length. A flow cytometric analysis of an EdU-labeling time course is shown. Roots of 3-day old seedlings were pulse-labeled with $25 \mathrm{QM}$ EdU for 5, 10, and $20 \mathrm{~min}$. Nuclei were isolated from the terminal $0-1 \mathrm{~mm}$ of fixed root segments and analyzed by flow cytometry. The S-phase nuclei with incorporated EdU (above dashed line) can be clearly distinguished from the non-replicating, unlabeled G1 and G2 nuclei populations (below dashed line). At 5 min, a detectable "arc" of EdU labeled nuclei has already formed, but the arc is further differentiated from the G1 and G2 populations after at least $10 \mathrm{~min}$. We chose a $20 \mathrm{~min}$ labeling time because of the clean separation of EdU-labeled nuclei from the unlabeled $\mathrm{G} 1$ and $\mathrm{G} 2$ populations for optimal sorting (see Note 1). 
Figure 4

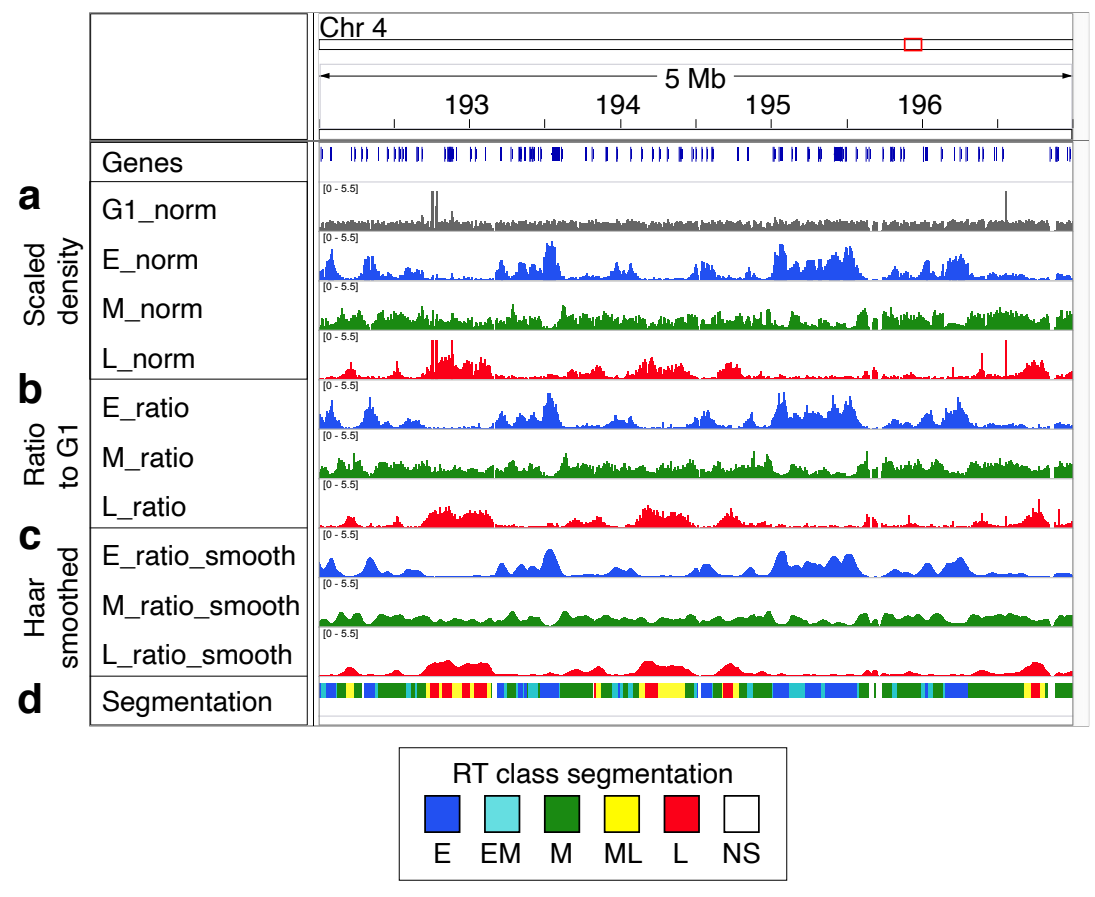

Fig. 4 Repliscan output files showing step by step analysis of Repli-seq data. (a-d) An example of Repliscan output for a 5-Mb region on chromosome 4 of the maize B73 genome, presented in the Integrated Genomics Viewer (IGV) genome browser. The small red rectangle located above the genome coordinates represents the location on chromosome 4 of the highlighted 5-Mb region. The dark blue tick marks represent annotated genes in B73 RefGen_v4. (a) Scaled read densities in 3-kb static bins (windows) were calculated for the G1 non-replicating control (grey) and the early (E), middle (M), and late (L) S-phase samples. The G1 control data are used to normalize for sequencing biases, read mappability variation in the reference genome, and possible differences in copy number. Notice the corresponding "spikes" of high signal in the tracks for the G1 control (grey) and the late S-phase sample (red). (b) For each S-phase sample, a ratio to G1 was calculated by dividing the scaled read density in each S-phase bin by the corresponding scaled read density in the G1 control. Notice the absence of "spikes" in the late S-phase track after this G1 normalization (see Note 22). (c) Haar wavelet smoothing was performed to remove noise without altering peak boundaries (section 3.11.7). (d) Each locus was classified (segmentation) with a replication time(s). The segmented RT classes represented are as follows: early (blue), early-mid (teal), middle (green), mid-late (yellow), late (red) and not-segmented (white) (see section 3.11.8). 


\section{Trim-galore-0.4.1}

Analysis Name:Trim-galore-0.4.1_analysis1

README

* Paired end Input fastq files

Parameters

Quality:

20

Phred64

$\checkmark$ fastqc

Adapter sequence to be trimmed:

Enter text

Note: If you want to use Adapter2, then this option requires 'paired' to be specified as well

Adapter2:

Enter text

stringency:

1

Error rate:

0.1

Compress the output file with gzip.

Do not compress the output file with gzip Length:

20

No report file

Clip R1:

\section{Enter text}

Clip R2 (Paired-end reads only):

Enter text

3' Clip R1:

Enter text

3' Clip R2 (Paired-end reads only):

\section{Enter text}

RRBS-specific options (MspI digested material)

Resource Requirements

Create Quick Launch

Launch Analysis

Fig. S1 Trim Galore! parameter window. The default parameters are entered, as well the fastqc checkbox selected to generate the FASTQC report. Any known Illumina adapters and index sequences should be entered in the Adapter sequence to be trimmed textbox. Trim Galore! will autodetect adapters if this parameter is left blank. 
Alignment options

Minimum seed length:

19

Bandwidth for banded alignment:

100

Off-diagonal X-dropoff:

100

Look for internal seeds inside a seed longer than $\{-k\} *$ FLOAT [1.5]:

\section{5}

Skip mate rescue

Skip pairing; mate rescue performed unless disabled above

Score for a sequence match:

1

Penalty for a mismatch:

4

Gap open penalties for deletions and insertions:

6

Gap extension penalty; a gap of size $k$ cost ' $\{-O\}+\{-E\}^{*} k^{\prime}$ :

1

Penalty for clipping:

5

Penalty for an unpaired read pair:

17

\section{Output options}

Minimum score to output:

30

Output all alignments for SE or unpaired PE

Append FASTA/FASTQ comment to SAM output

Use soft clipping for supplementary alignments

mark shorter split hits as secondary

Resource Requirements

Create Quick Launch

Launch Analysis

Fig. S2 BWA-MEM parameters and output options windows. The BWA-MEM default parameters (a) and default output options (b) are auto-populated. 
Figure S3

a

\section{Csamtools view 1.9}

Analysis Name:samtools_view_1.9_analysis1

\section{* Inputs}

Parameters

$\checkmark$ Use MAPQ filtering

MAPQ Value to filter:

10

Filter reads that are unmapped and non-primary mapped (SAM flag: 0x104)

$\checkmark$ Keep only read properly paired (SAM flag: 0x2)

$\checkmark$ Remove reads that are supplementary alignments (SAM flag: 0x800)

Plot distribution of MAPQ

b

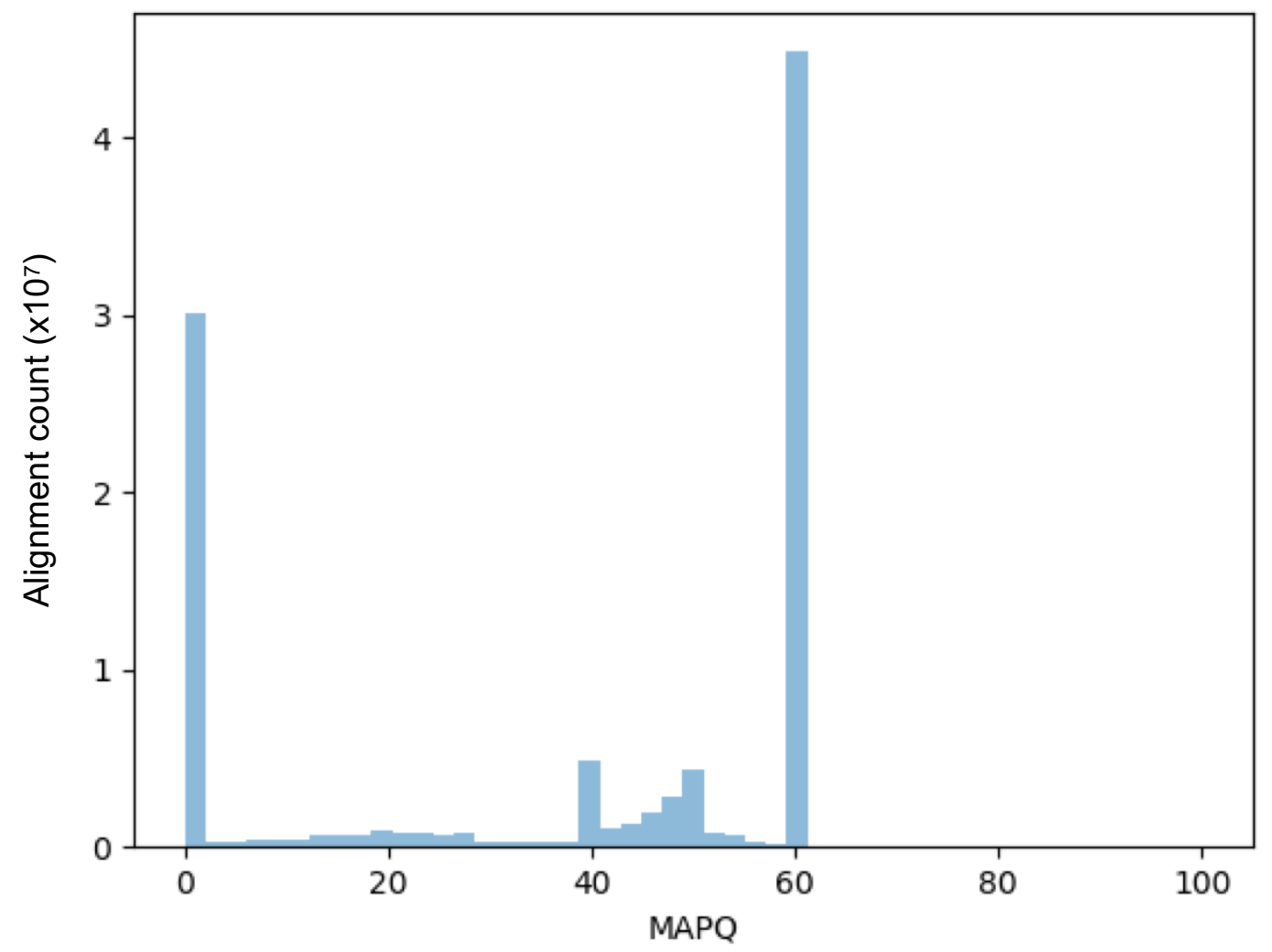

Fig. S3 SAMtools view parameters window. (a) The SAMtools view app recommended parameters are selected. (b) The output from Plot distribution of MAPQ is a plot of the mapping quality (MAPQ) distribution found in the alignment file before filtering. 


\begin{tabular}{|l|r|}
\hline C Picard-MarkDuplicates-2.7.1 & - \\
\hline Analysis Name:Picard-MarkDuplicates-2.7.1_analysis1 & \\
\hline License agreement. Please use this carefully before using this App!!!! & - \\
\hline README & - \\
\hline * Input & \\
\hline * Output(s) & (1) \\
\hline Parameters & (1) \\
\hline Validation stringency: & - \\
\hline LENIENT & \\
\hline$\checkmark$ Remove duplicates & \\
\hline Resource Requirements & Launch Analysis \\
\hline
\end{tabular}

Fig. S4 Picard MarkDuplicates parameter window. The recommended parameters for marking and removing duplicate read alignments. 


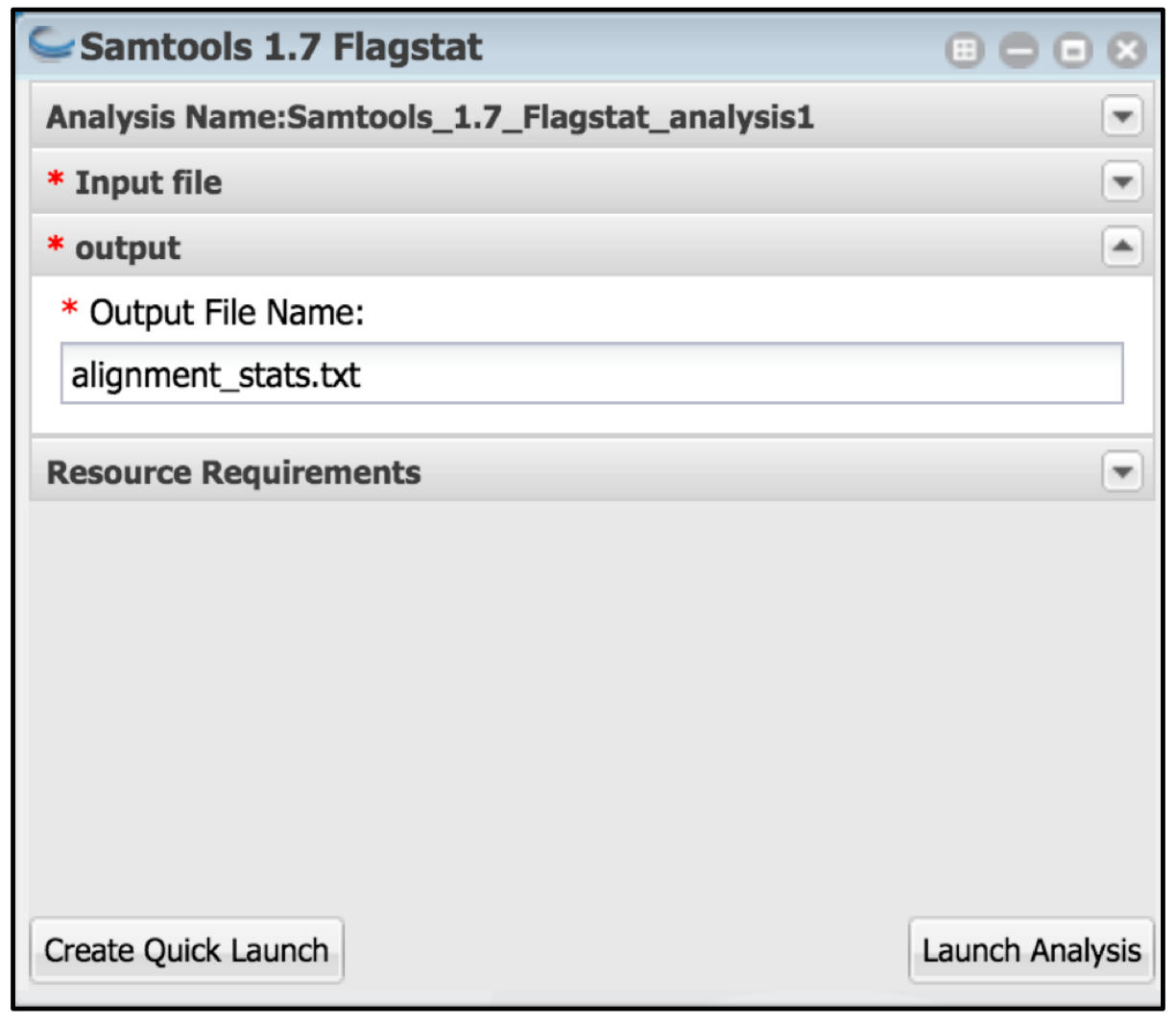

Fig. S5 SAMtools Flagstat output window. SAMtools Flagstat generates mapping statistics outputted as a nameable .txt file. 


\section{* Inputs}

* Parameters

* bins:

3000

removeoutliers

$\log 10$

* Correlation type:

pearson

b

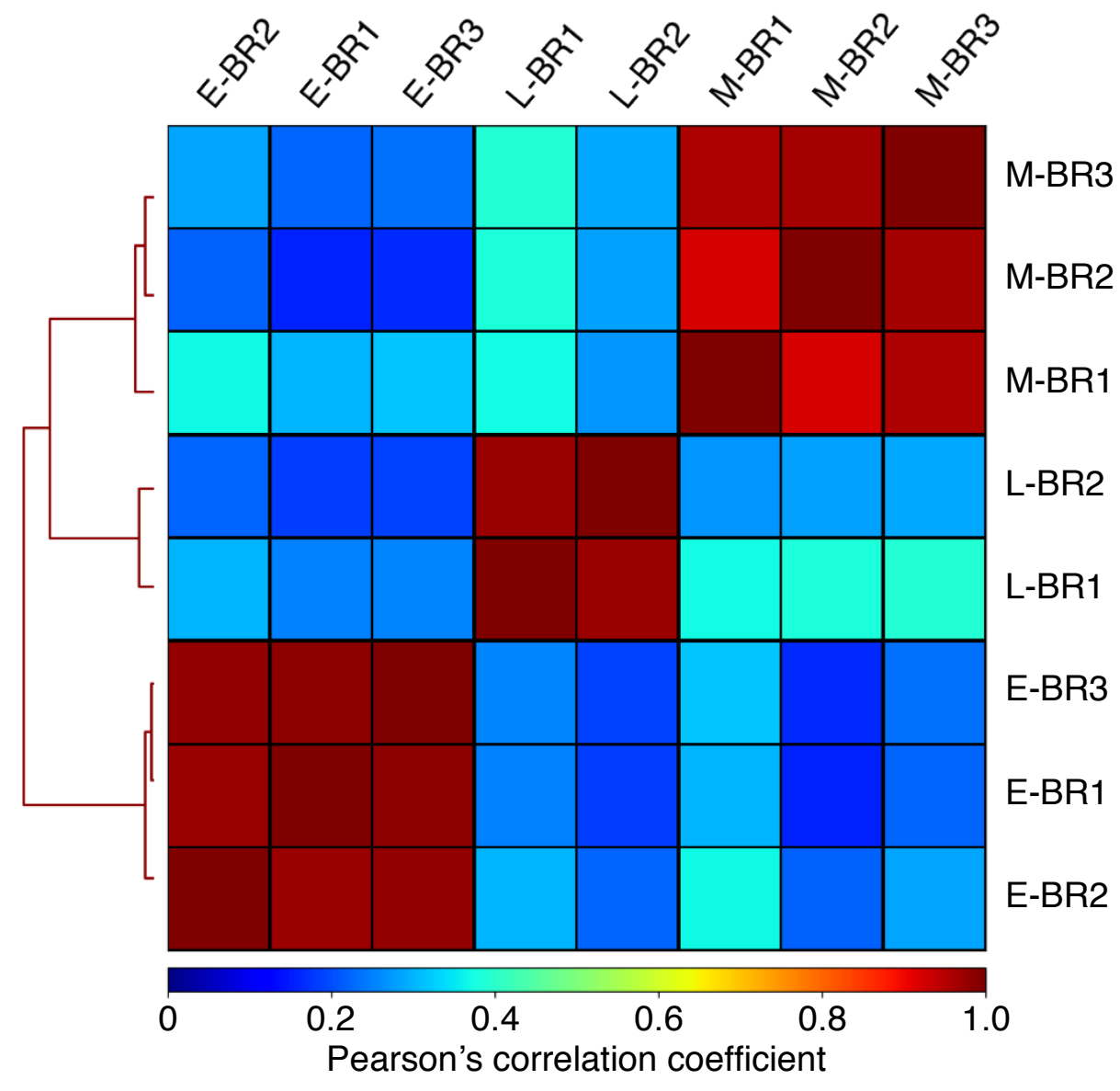

Fig. S6 DeepTools multiBamSummary parameters window.

(a) The recommended Correlation type is selected. A desired static bin size (bins in base pairs) should also be selected. (b) The Pearson correlation coefficients between individual biological replicates for early, middle and late $S$ samples represented as a heatmap with a hierarchical clustering dendrogram. 


\begin{tabular}{|c|c|}
\hline$\subseteq$ repliscan 0.1.0 & $B-0 \times$ \\
\hline Parameters & $\Delta$ \\
\hline value: & (i) \\
\hline 1 & $\hat{v}$ \\
\hline classifier: & (i) \\
\hline proportion & $\checkmark$ \\
\hline $\begin{array}{l}\checkmark \text { plot } \\
\text { threshold: }\end{array}$ & (i) \\
\hline auto & $\mathbf{v}$ \\
\hline percent: & (i) \\
\hline 2 & $\hat{\mathbf{v}}$ \\
\hline remove: & (1) \\
\hline norm & $\mathbf{v}$ \\
\hline scope: & (i) \\
\hline chromosome & $\checkmark$ \\
\hline percentil cutoff: & (i) \\
\hline $2.5-97.5$ & 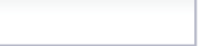 \\
\hline $\begin{array}{l}\square \log \\
\text { aggregate: }\end{array}$ & (i) \\
\hline sum & $\checkmark$ \\
\hline Haar smoothing level: & (i) \\
\hline 3 & $\hat{v}$ \\
\hline Analysis bin size in base pairs (Default: 1000 ): & (i) \\
\hline 1000 & $\hat{v}$ \\
\hline Create Quick Launch & Launch Analysis \\
\hline
\end{tabular}

Fig. S7 Repliscan default parameters. The Repliscan app default parameters are auto-populated. Select the plot checkbox to generate additional output files (see Table 2 and section 3.11.9). 


\begin{tabular}{|c|c|}
\hline Crepliscan 0.1.0 & $\boxplus \ominus \square \times$ \\
\hline Parameters & $\Delta$ \\
\hline value: & (1) \\
\hline 1 & $\hat{\mathbf{v}}$ \\
\hline classifier: & (1) \\
\hline proportion & $\mathbf{v}$ \\
\hline $\begin{array}{l}\checkmark \text { plot } \\
\text { threshold: }\end{array}$ & (1) \\
\hline auto & $\mathbf{v}$ \\
\hline percent: & (1) \\
\hline Enter a value & $\hat{v}$ \\
\hline remove: & (1) \\
\hline percentile & $\checkmark$ \\
\hline scope: & (1) \\
\hline chromosome & $\mathbf{v}$ \\
\hline percentil cutoff: & (1) \\
\hline \multicolumn{2}{|l|}{$1.0-100$} \\
\hline $\begin{array}{l}\square \text { log } \\
\text { aggregate: }\end{array}$ & (1) \\
\hline sum & $\mathbf{v}$ \\
\hline Haar smoothing level: & (1) \\
\hline 3 & $\hat{v}$ \\
\hline Analysis bin size in base pairs (Default: 1000 ): & (1) \\
\hline 3000 & $\hat{v}$ \\
\hline Create Quick Launch & Launch Analysis \\
\hline
\end{tabular}

Fig. S8 Repliscan recommended parameters for maize B73 test dataset. For the maize test dataset, which has been filtered to remove alignments with $M A P Q<10$ and randomly downsampled, we recommend adjusting the Analysis bin size in base pairs as well as the remove parameter with the dependent parameter percentile cutoff. These setting adjustments are shown in the parameter window. See Note 21 and 22 for a detailed description. 
Figure S9

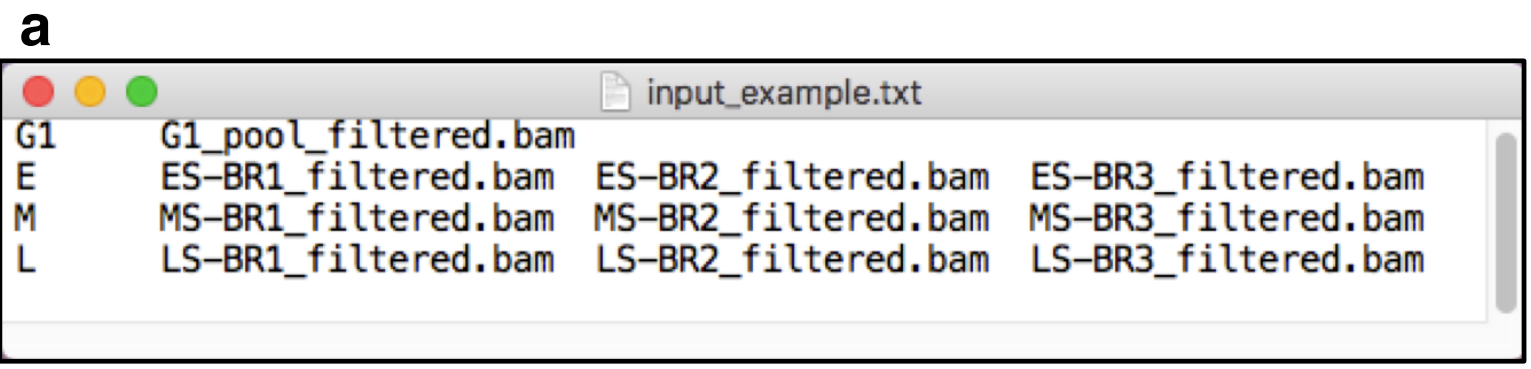

\section{b}

\begin{tabular}{|c|c|c|}
\hline \multicolumn{3}{|c|}{ Crepliscan $\mathbf{0 . 1 . 0}$} \\
\hline \multicolumn{3}{|c|}{ Analysis Name:repliscan_0.1.0_analysis1 } \\
\hline \multicolumn{3}{|c|}{ * Inputs } \\
\hline \multicolumn{3}{|c|}{ * Reference genome in Fasta format: } \\
\hline 5 & /iplant/home/shared/iplantcollaborative/genomeservices/builds/1.0.0/24_77/Zea.AGPv4/de_support/genome.fa & Browse \\
\hline \multicolumn{2}{|c|}{ * File with list of bams: } & (1) \\
\hline 5 & /iplant/home/shared/iplantcollaborative/example_data/repliscan/input_ds.txt & Browse \\
\hline \multicolumn{2}{|c|}{ * Directory with bam files: } & (1) \\
\hline 5 & /iplant/home/shared/iplantcollaborative/example_data/repliscan & Browse \\
\hline \multicolumn{2}{|c|}{ Parameters } & - \\
\hline \multicolumn{2}{|c|}{ Create Quick Launch } & ch Analysis \\
\hline
\end{tabular}

Fig. S9 Repliscan input configuration example. (a) An input.txt configuration file is needed to assign individual files to analysis name labels. Individual files should be delimited by a tab. The name labels are used in output file naming and RT class segmentation naming. (b) Repliscan Inputs window requiring reference genome in Fasta format, a configuration file with a list of .bam files (input.txt), and a directory containing the .bam files. 
a

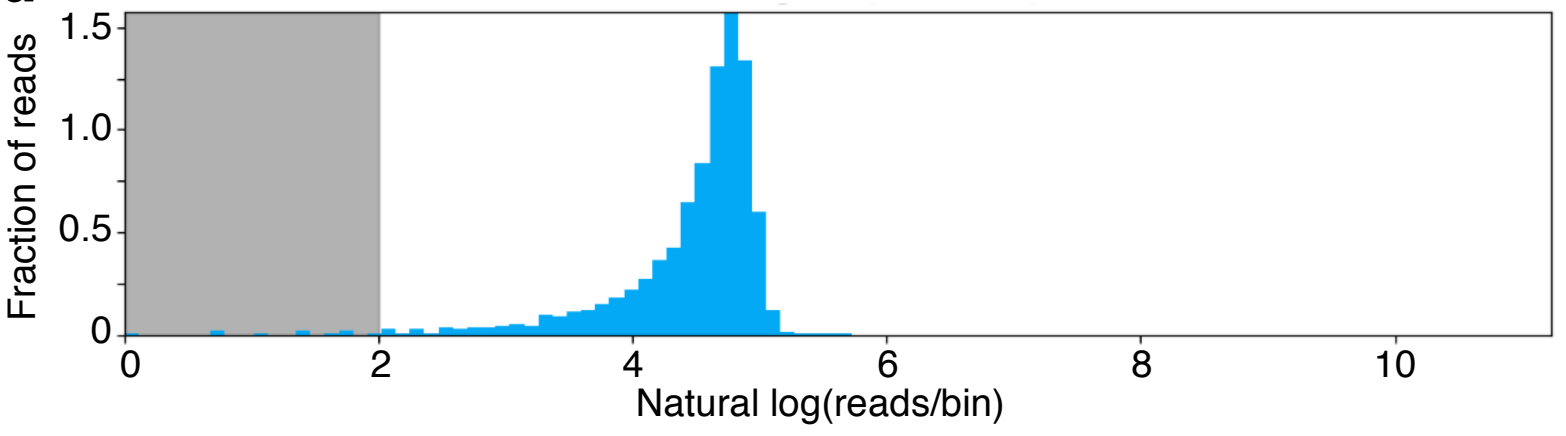

b

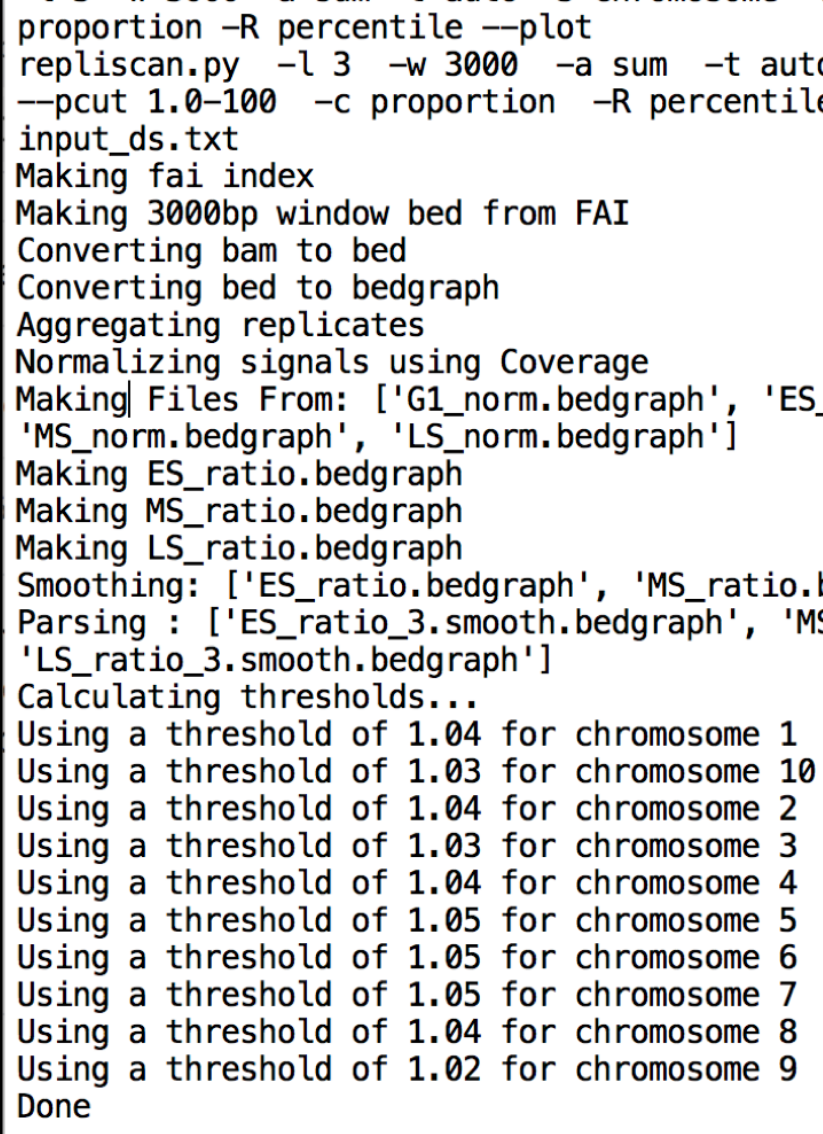

Fig. S10 Repliscan optional output example. Selecting the plot checkbox generates additional output files. (a) Included in these output files is a plot of the distribution of natural log transformed reads per bin in the G1 data and the selected cutoff (grey shaded area) from the remove and percentile cutoff parameters. For maize data that has been filtered to remove alignments with $M A P Q<10$, the distribution is negatively skewed (see Note 22). (b) An example of the .out file, which includes the parameter settings used and the auto-tuned RT class segmentation thresholds for individual chromosomes (see section 3.11.8). The plots shown represent the files from the maize B73 test dataset with recommended parameters (Fig. S8). 


\begin{tabular}{|l|l|}
\hline Analysis Name:repliscan_0.1.0_analysis1 & \\
\hline Analysis Name: & \\
\hline repliscan_0.1.0_analysis1 & \\
Comments: & Browse \\
\hline Select output folder: & \\
\hline /iplant/home/emwear/analyses & \\
\hline * Inputs & \\
\hline Parameters & \\
\hline Create Quick Launch & \\
\hline
\end{tabular}

Fig. S11 Analysis Name dropdown window example. Each app listed in the Repli-seq data analysis pipeline (Table 1) follows the same general organization. In the Analysis Name section, the specific name of the run and the location of the output folder can be modified. 